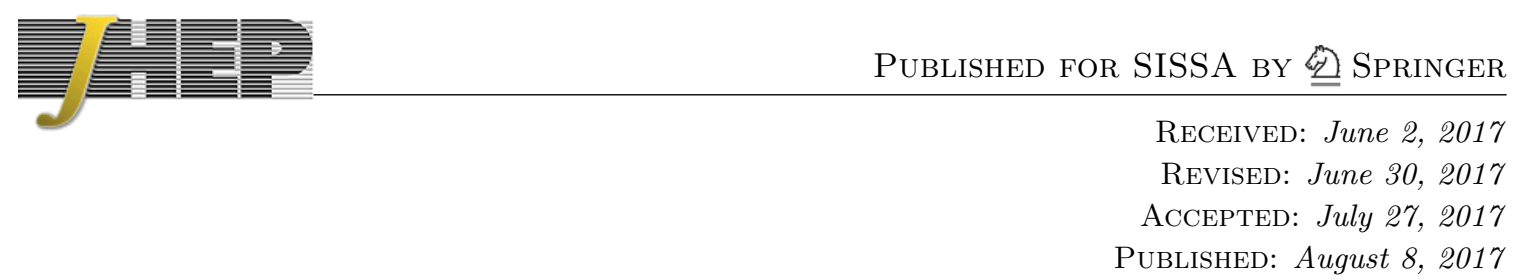

\title{
Lepton flavor changing Higgs decays in the littlest Higgs model with T-parity
}

\author{
Francisco del Aguila, ${ }^{a}$ Lluis Ametller, ${ }^{b}$ Jose Ignacio Illana, ${ }^{a}$ Jose Santiago, ${ }^{a}$ \\ Pere Talavera $^{c}$ and Roberto Vega-Morales ${ }^{a}$ \\ ${ }^{a}$ CAFPE and Departamento de Física Teórica y del Cosmos, Universidad \\ de Granada, E-18071 Granada, Spain \\ ${ }^{b}$ Departament de Física, Universitat Politècnica de Catalunya, \\ E-08034 Barcelona, Spain \\ ${ }^{c}$ Institut de Ciencies del Cosmos, Universitat de Barcelona (IEEC-UB), \\ Marti i Franques 1, Barcelona 08028, Spain \\ E-mail: faguila@ugr.es, lluis.ametller@upc.edu, jillana@ugr.es, \\ jsantiago@ugr.es, ptalavera@iese.edu, rvegamorales@ugr.es
}

ABSTRACT: We calculate loop induced lepton flavor violating Higgs decays in the Littlest Higgs model with T-parity. We find that a finite amplitude is obtained only when all contributions from the T-odd lepton sector are included. This is in contrast to lepton flavor violating processes mediated by gauge bosons where the partners of the right-handed mirror leptons can be decoupled from the spectrum. These partners are necessary to cancel the divergence in the Higgs mass introduced by the mirror leptons but are otherwise unnecessary and assumed to be decoupled in previous phenomenological studies. Furthermore, as we emphasize, including the partner leptons in the spectrum also introduces a new source of lepton flavor violation via their couplings to the physical pseudo-Goldstone electroweak triplet scalar. Although this extra source also affects lepton flavor changing gauge transitions, it decouples from these amplitudes in the limit of heavy mass for the partner leptons. We find that the corresponding Higgs branching ratio into taus and muons can be as large as $\sim 0.2 \times 10^{-6}$ for T-odd masses of the order a few $\mathrm{TeV}$, a demanding challenge even for the high luminosity LHC.

Keywords: Beyond Standard Model, Higgs Physics, Effective Field Theories

ARXIV EPRINT: 1705.08827 


\section{Contents}

1 Introduction 1

2 Reviewing the LHT 3

2.1 Mass eigenfields and Feynman rules $\quad 9$

2.1.1 Mass eigenfields 9

$\begin{array}{lll}2.1 .2 & \text { Feynman rules } & 12\end{array}$

3 Higgs coupling to a pair of different charged leptons at one loop in the LHT

3.1 One-loop contribution of T-odd particles to $h \rightarrow \bar{\ell} \ell^{\prime}$ in the LHT 15

3.2 New sources of flavor violation contributing to $h \rightarrow \bar{\ell} \ell^{\prime}$ in the LHT 17

4 Model dependent limits on the Higgs coupling to a pair of different lep$\begin{array}{ll}\text { tons in the LHT } & 19\end{array}$

$\begin{array}{llr}5 & \text { Summary and conclusions } & 20\end{array}$

$\begin{array}{ll}\text { A Expressions for the amplitudes } & 21\end{array}$

\section{Introduction}

The discovery at the LHC [1, 2] of a Standard Model (SM) like Higgs [3-5] appears to have settled the nature of the electroweak symmetry breaking (EWSB) mechanism, but the lack of any signs of physics beyond the SM still leaves unsolved the apparent fine tuning problem of the electroweak scale. While lack of observed new physics strongly constrains the most natural models addressing the hierarchy problem, room still exists for a solution at $\sim \mathrm{TeV}$ which is perhaps observable at the LHC.

Although supersymmetry is the most well known solution, Little Higgs models [6] also offer an elegant solution and will be further tested in the coming years at the LHC. (For a review see refs. [7-9].) A popular, phenomenologically viable realization of this class of models is the Littlest Higgs model with T-parity [10-12] (LHT), which is protected at tree level against constraints from electroweak precision $[13,14]$ data (EWPD). Restricting ourselves to 'non-linear' Callan-Coleman-Wess-Zumino [15] constructions [12, 16], the LHT possesses a global SU(5) symmetry broken spontaneously at a scale $f \sim \mathrm{TeV}$ down to an $\mathrm{SO}(5)$ subgroup. This gives rise to a Goldstone sector containing the SM Higgs doublet as well as an electroweak triplet with zero vacuum expectation value $(v e v){ }^{1}$ Two different

\footnotetext{
${ }^{1}$ This is true only if T-parity remains exact, but a tiny vev along the neutral direction of this pseudoGoldstone scalar triplet can be invoked to give very small Majorana masses to the SM neutrinos [17].
} 
$\mathrm{SU}(2) \times \mathrm{U}(1)$ subgroups of $\mathrm{SU}(5)$ are gauged with equal strength and broken spontaneously down to a diagonal subgroup which is identified with the SM gauge group, while the broken gauge symmetries lead to a set of massive vector bosons with mass $\sim f$. Utilizing a $Z_{2}$ automorphism inherited from the global symmetry breaking structure, a 'T-parity' can be defined for the gauge sector under which all SM particles are even and with mass scale $v \sim 246 \mathrm{GeV}$ and (almost) all new particles are odd with mass scale $f$.

There have been many phenomenological studies of this type of Little Higgs models [1830]. A number of these have examined specifically the possibility of lepton flavor violation (LFV) in processes at LEP and LHC or other experiments $[18,26-29] .^{2}$ The underlying source of this LFV comes from the Yukawa sectors responsible for generating the masses of the light SM leptons and the heavy T-odd 'mirror' leptons. In particular, it arises from the rotations in flavor space which are necessary to diagonalize their mass matrices. However, as we emphasize in this work, this is not the only possible source of LFV in the LHT. Apart from the T-odd mirror leptons, which acquire a mass $\sim f$ after the $\mathrm{SU}(5)$ breaking, there are additional T-odd lepton doublets (also vector-like) required to maintain the $\mathrm{SO}(5)$ global symmetry protecting the Higgs mass from dangerous divergences [11]. These do not acquire a mass from the SU(5) breaking and are often not necessary for understanding the essential aspects of the LHT or much of its phenomenology. Thus, in virtually all phenomenological studies of LFV in the LHT, it is assumed that these additional leptons completing the $\mathrm{SO}(5)$ right-handed representations are decoupled from the spectrum, though light enough to not reintroduce a fine tuning in the Higgs mass for which they serve as a cutoff at two loops [11]. However, as we examine more closely below, such a decoupling does not hold when dealing with Higgs decays. Moreover, since they do not acquire a mass from the same mechanism which generates masses for the mirror leptons, these partner leptons must be given separate masses. These masses, which softly break the global SO(5), will themselves carry generation indices and introduce additional flavor structure in the Yukawa sector.

The rotations of these additional lepton fields, required to diagonalize their mass matrix, are independent of the previous rotations needed to diagonalize the SM and mirror lepton masses and introduce a potential new source of LFV. In this sense, previous studies which assume these partner leptons are heavy are not exhaustive. Furthermore, since the new contributions from the heavy partner leptons are necessary to render LFV Higgs decay amplitudes finite, any previous estimates of these decays which neglect them must be recomputed to give an unambiguous prediction. In contrast, LFV effects mediated by photons or $Z$ bosons which explicitly depend on these new rotations become negligible in the limit of heavy partner leptons and in particular, there is no divergence introduced when the partner leptons are neglected. So while LFV effects, in $h \rightarrow \tau \mu$ for instance, from the partner leptons can be sizable even when they are heavy compared to the $\mathrm{SU}(5)$ breaking scale $f$, they do decouple in processes mediated by gauge bosons such as $Z \rightarrow \tau \mu$. This implies that $h \rightarrow \tau \mu$ decays, for which constraints from LHC are weaker [35], could be significantly larger than naively expected given constraints on LFV from processes mediated by photons or $Z$ bosons.

\footnotetext{
${ }^{2}$ Similar studies for the Simplest Little Higgs model can be found in refs. [31-34], and references therein.
} 
We compute the one-loop contributions to $h \rightarrow \tau \mu$ decays and show that the T-odd particles sum to a finite amplitude, but only once all T-odd leptons are included. We emphasize this here because the contributions from the mirror leptons, which are not decoupled in studies of $\gamma$ and $Z$ mediated LFV transitions, are not finite by themselves in $h \rightarrow \tau \mu .^{3}$ This is in contrast to $Z \rightarrow \tau \mu$ for example where they are indeed finite. Furthermore, as we examine below, including the partner leptons in the spectrum necessarily introduces new couplings to the physical pseudo-Goldstone electroweak triplet scalar which will enter into LFV amplitudes and which depend explicitly on the rotations of the heavy partner lepton fields needed to diagonalize their mass matrices. Although this extra source also enters in gauge boson mediated LFV amplitudes, its effects decouple in the limit of heavy mass for the partner leptons. For $h \rightarrow \tau \mu$ their contribution, through which the new LFV source enters, does not decouple in the limit of heavy partner lepton masses and instead grows logarithmically. These logarithmic corrections are indicative of a soft breaking of the global $\mathrm{SO}(5)$ by the partner lepton masses and are related to corrections to the Higgs mass. Therefore, the partner lepton masses cannot be taken arbitrarily large without reintroducing a fine tuning problem in the Higgs mass at two loops.

To be self contained and to fix our notation we review in the next section the LHT and provide the new set of Feynman rules necessary for computing LFV Higgs decays, including the couplings involving the heavy partner leptons. The interested reader can go directly to section 3 where we present the calculation of the $h \rightarrow \bar{\ell} \ell^{\prime}$ amplitude. For completeness, the detailed expression of the contribution independent of the new LFV source in the $h \rightarrow \bar{\ell} \ell^{\prime}$ amplitude is gathered in the appendix. Section 4 is devoted to a short phenomenological discussion, including the effect of the proper redefinition of the final mass eigenstates. We also point out the main parametric limits for the $h \rightarrow \tau \mu$ branching ratio, which can be raised up to $\sim 0.2 \times 10^{-6}$ in the limit of large mirror and partner lepton masses, becoming a demanding challenge even for the high luminosity LHC. A detailed phenomenological discussion will be presented elsewhere [38], together with a reanalysis of LFV processes mediated by neutral gauge bosons which includes the new contributions from the right handed partner leptons. The final section is devoted to our conclusions.

\section{Reviewing the LHT}

Here we summarize the LHT to fix our notation and provide the new Feynman rules necessary for the one loop calculation of the $h \rightarrow \tau \mu$ amplitude. Since the amplitude is higher order in an operator expansion in SM fields it is in general suppressed by $v^{2} / f^{2}$ and clearly decouples when $f$ goes to infinity. Thus, in general the Feynman rules involving the heavy T-odd particles must also be worked out to this order. Though we attempt to be as self-contained as possible, we closely follow the presentation in refs. [26, 39].

The LHT is a non-linear $\sigma$ model with a $\mathrm{SU}(5)$ global symmetry broken down to $\mathrm{SO}(5)$ by the vev of a $5 \times 5$ symmetric tensor,

$$
\Sigma_{0}=\left(\begin{array}{ccc}
\mathbf{0}_{2 \times 2} & 0 & \mathbf{1}_{2 \times 2} \\
0 & 1 & 0 \\
\mathbf{1}_{2 \times 2} & 0 & \mathbf{0}_{2 \times 2}
\end{array}\right) .
$$

\footnotetext{
${ }^{3}$ Against what is stated in refs. [36, 37].
} 
The 10 unbroken $\mathrm{SU}(5)$ generators $T^{a}$, which leave invariant $\Sigma_{0}$ and hence satisfy the equality $T^{a} \Sigma_{0}+\Sigma_{0}\left(T^{a}\right)^{T}=0$, generate the $\mathrm{SO}(5)$ algebra; whereas the 14 broken $\mathrm{SU}(5)$ generators $X^{a}$, which fulfill the relation $X^{a} \Sigma_{0}-\Sigma_{0}\left(X^{a}\right)^{T}=0$, expand the Goldstone matrix $\Pi=\pi^{a} X^{a}$ parameterizing the $5 \times 5$ symmetric tensor

$$
\Sigma(x)=\mathrm{e}^{i \Pi / f} \Sigma_{0} \mathrm{e}^{i \Pi^{T} / f}=\mathrm{e}^{2 i \Pi / f} \Sigma_{0},
$$

with $f$ the scale of new physics (NP). It is important to note that this breaking fixes the embedding of $\mathrm{SO}(5)$ in $\mathrm{SU}(5)$, with the fundamental representation of $\mathrm{SU}(5)$ reducing to the defining (real) representation of $\mathrm{SO}(5)$, both of dimension five.

$\mathrm{An} \mathrm{SU}(2)_{1} \times \mathrm{SU}(2)_{2} \times \mathrm{U}(1)_{1} \times \mathrm{U}(1)_{2}$ subgroup of the $\mathrm{SU}(5)$ is gauged and generated by

$$
\begin{aligned}
Q_{1}^{a}=\frac{1}{2}\left(\begin{array}{ccc}
\sigma^{a} & 0 & 0 \\
0 & 0 & 0 \\
0 & 0 & \mathbf{0}_{2 \times 2}
\end{array}\right), & Y_{1}=\frac{1}{10} \operatorname{diag}(3,3,-2,-2,-2), \\
Q_{2}^{a}=\frac{1}{2}\left(\begin{array}{ccc}
\mathbf{0}_{2 \times 2} & 0 & 0 \\
0 & 0 & 0 \\
0 & 0 & -\sigma^{a *}
\end{array}\right), & Y_{2}=\frac{1}{10} \operatorname{diag}(2,2,2,-3,-3),
\end{aligned}
$$

with $\sigma^{a}$ the three Pauli matrices. The vev in eq. (2.1) breaks this gauge group down to the SM gauge group $\mathrm{SU}(2)_{L} \times \mathrm{U}(1)_{Y}$, generated by the combinations $\left\{Q_{1}^{a}+Q_{2}^{a}, Y_{1}+Y_{2}\right\} \subset\left\{T^{a}\right\}$. The orthogonal combinations are a subset of the broken generators, $\left\{Q_{1}^{a}-Q_{2}^{a}, Y_{1}-Y_{2}\right\} \subset$ $\left\{X^{a}\right\}$. Thus, the Goldstone matrix

$$
\Pi=\left(\begin{array}{ccccc}
-\frac{\omega^{0}}{2}-\frac{\eta}{\sqrt{20}} & -\frac{\omega^{+}}{\sqrt{2}} & -i \frac{\pi^{+}}{\sqrt{2}} & -i \Phi^{++} & -i \frac{\Phi^{+}}{\sqrt{2}} \\
-\frac{\omega^{-}}{\sqrt{2}} & \frac{\omega^{0}}{2}-\frac{\eta}{\sqrt{20}} & \frac{v+h+i \pi^{0}}{2} & -i \frac{\Phi^{+}}{\sqrt{2}} & \frac{-i \Phi^{0}+\Phi^{P}}{\sqrt{2}} \\
i \frac{\pi^{-}}{\sqrt{2}} & \frac{v+h-i \pi^{0}}{2} & \sqrt{\frac{4}{5} \eta} & -i \frac{\pi^{+}}{\sqrt{2}} & \frac{v+h+i \pi^{0}}{2} \\
i \Phi^{--} & i \frac{\Phi^{-}}{\sqrt{2}} & i \frac{\pi^{-}}{\sqrt{2}} & -\frac{\omega^{0}}{2}-\frac{\eta}{\sqrt{20}} & -\frac{\omega^{-}}{\sqrt{2}} \\
i \frac{\Phi^{-}}{\sqrt{2}} & \frac{i \Phi^{0}+\Phi^{P}}{\sqrt{2}} & \frac{v+h-i \pi^{0}}{2} & -\frac{\omega^{+}}{\sqrt{2}} & \frac{\omega^{0}}{2}-\frac{\eta}{\sqrt{20}}
\end{array}\right)
$$

decomposes into the SM Higgs doublet $\phi^{T}=\left(-i \pi^{+},\left(v+h+i \pi^{0}\right) / \sqrt{2}\right)^{T}$, a complex $\mathrm{SU}(2)_{L}$ triplet $\Phi$, and the longitudinal modes of the heavy gauge fields $\omega^{ \pm}, \omega^{0}$ and $\eta{ }^{4}$

Gauge Lagrangian. T-parity is introduced to make the new heavy particles T-odd, keeping the SM fields T-even (invariant). Its action on the gauge fields $G_{i}$ exchanges the two gauge groups $\mathrm{SU}(2)_{1} \times \mathrm{U}(1)_{1}$ and $\mathrm{SU}(2)_{2} \times \mathrm{U}(1)_{2}$,

$$
G_{1} \stackrel{\mathrm{T}}{\longleftrightarrow} G_{2}
$$

\footnotetext{
${ }^{4}$ In the following we use for the SM fields and couplings the conventions in ref. [40]. In particular, $\phi^{+}=-i \pi^{+}, \phi^{0}=\pi^{0}$.
} 
T-parity then requires the gauge couplings to be equal leading to the gauge Lagrangian

$$
\mathcal{L}_{G}=\sum_{j=1}^{2}\left[-\frac{1}{2} \operatorname{Tr}\left(\widetilde{W}_{j \mu \nu} \widetilde{W}_{j}^{\mu \nu}\right)-\frac{1}{4} B_{j \mu \nu} B_{j}^{\mu \nu}\right],
$$

which is $\mathrm{T}$ parity and gauge invariant where we have defined

$$
\widetilde{W}_{j \mu}=W_{j \mu}^{a} Q_{j}^{a}, \quad \widetilde{W}_{j \mu \nu}=\partial_{\mu} \widetilde{W}_{j \nu}-\partial_{\nu} \widetilde{W}_{j \mu}-i g\left[\widetilde{W}_{j \mu}, \widetilde{W}_{j \nu}\right], \quad B_{j \mu \nu}=\partial_{\mu} B_{j \nu}-\partial_{\nu} B_{j \mu}
$$

and repeated indices are understood to be summed. The SM gauge bosons are the T-even combinations multiplying the unbroken gauge generators,

$$
W^{ \pm}=\frac{1}{2}\left[\left(W_{1}^{1}+W_{2}^{1}\right) \mp i\left(W_{1}^{2}+W_{2}^{2}\right)\right], \quad W^{3}=\frac{W_{1}^{3}+W_{2}^{3}}{\sqrt{2}}, \quad B=\frac{B_{1}+B_{2}}{\sqrt{2}} ;
$$

whereas the heavy gauge bosons are the T-odd combinations

$$
W_{H}^{ \pm}=\frac{1}{2}\left[\left(W_{1}^{1}-W_{2}^{1}\right) \mp i\left(W_{1}^{2}-W_{2}^{2}\right)\right], \quad W_{H}^{3}=\frac{W_{1}^{3}-W_{2}^{3}}{\sqrt{2}}, \quad B_{H}=\frac{B_{1}-B_{2}}{\sqrt{2}} .
$$

Scalar Lagrangian. Likewise, to keep the SM Higgs doublet T-even and make the remaining Goldstone fields T-odd, we define the $\mathrm{T}$ action on the scalar fields

$$
\Pi \stackrel{\mathrm{T}}{\longrightarrow}-\Omega \Pi \Omega, \quad \Omega=\operatorname{diag}(-1,-1,1,-1,-1),
$$

where $\Omega$ is an element of the center of the gauge group commuting with $\Sigma_{0}$ but not with the full global symmetry. ${ }^{5}$ Hence,

$$
\Sigma \stackrel{\mathrm{T}}{\longrightarrow} \widetilde{\Sigma}=\Omega \Sigma_{0} \Sigma^{\dagger} \Sigma_{0} \Omega
$$

while the scalar kinetic Lagrangian is given by

$$
\mathcal{L}_{S}=\frac{f^{2}}{8} \operatorname{Tr}\left[\left(D_{\mu} \Sigma\right)^{\dagger}\left(D^{\mu} \Sigma\right)\right]
$$

with the covariant derivative defined as

$$
D_{\mu} \Sigma=\partial_{\mu} \Sigma-\sqrt{2} i \sum_{j=1}^{2}\left[g W_{j \mu}^{a}\left(Q_{j}^{a} \Sigma+\Sigma Q_{j}^{a T}\right)-g^{\prime} B_{j \mu}\left(Y_{j} \Sigma+\Sigma Y_{j}^{T}\right)\right] .
$$

Fermionic Lagrangian. Implementing T-parity in the fermionic sector of the Littlest Higgs model is less straightforward. Three types of couplings are needed to give masses to all leptons in the model. It is the misalignment between these couplings which results in the two sources of LFV which are beyond the SM. ${ }^{6}$

\footnotetext{
${ }^{5}$ Note that we have reversed the sign of $\Omega$ as compared to the literature, to make it a group element.

${ }^{6} \mathrm{LFV}$ is highly suppressed in the SM due to the tiny neutrino mass which we take them as massless throughout this work.
} 
Following refs. $[11,12]$ for each SM left-handed $(L)$ lepton doublet we introduce two incomplete $\mathrm{SU}(5)$ multiplets in fundamental representations:

$$
\Psi_{1}=\left(\begin{array}{c}
-i \sigma^{2} l_{1 L} \\
0 \\
0
\end{array}\right), \quad \Psi_{2}=\left(\begin{array}{c}
0 \\
0 \\
-i \sigma^{2} l_{2 L}
\end{array}\right)
$$

where $l_{r L}=\left(\begin{array}{c}\nu_{r L} \\ \ell_{r L}\end{array}\right), r=1,2 ;$ and

$$
\Psi_{1} \longrightarrow V^{*} \Psi_{1}, \quad \Psi_{2} \longrightarrow V \Psi_{2}
$$

under the $\mathrm{SU}(5)$ transformation $V$. T-parity is then defined

$$
\Psi_{1} \stackrel{\mathrm{T}}{\longleftrightarrow} \Omega \Sigma_{0} \Psi_{2}
$$

where the T-even combination is given by $\Psi_{1}+\Omega \Sigma_{0} \Psi_{2}$ and identified with the SM lefthanded lepton doublet, up to the proper normalization. The orthogonal combination $\Psi_{1}-\Omega \Sigma_{0} \Psi_{2}$ defines a second left-handed lepton doublet which is T-odd (see eqs. (2.13) and (2.15)), and which must be paired to a right-handed $(R)$ 'mirror' lepton doublet $l_{H R}$ in order to obtain a large (vector-like) mass of $\mathcal{O}(f)$. ( $H$ stands for heavy.)

The mirror leptons introduce divergences into the Higgs mass as well as the $h \rightarrow \tau \mu$ amplitude which we examine below. These divergences must be cancelled by introducing additional right handed 'partner' leptons $\tilde{\psi}_{R}$, along with $l_{H R}$ and an additional singlet $\chi_{R}$, to form a complete $\mathrm{SO}(5)$ multiplet which we define as:

$$
\Psi_{R}=\left(\begin{array}{c}
\tilde{\psi}_{R} \\
\chi_{R} \\
-i \sigma^{2} l_{H R}
\end{array}\right), \quad \Psi_{R} \longrightarrow U \Psi_{R} .
$$

$\Psi_{R}$ is a T-eigenvector

$$
\Psi_{R} \stackrel{\mathrm{T}}{\longrightarrow} \Omega \Psi_{R}
$$

The mirror leptons obtain their masses through the non-linear Yukawa Lagrangian:

$$
\mathcal{L}_{Y_{H}}=-\kappa f\left(\bar{\Psi}_{2} \xi+\bar{\Psi}_{1} \Sigma_{0} \xi^{\dagger}\right) \Psi_{R}+\text { h.c. },
$$

where $\xi=\mathrm{e}^{i \Pi / f}$. This is T-invariant, since eq. (2.9) implies

$$
\xi \stackrel{\mathrm{T}}{\rightarrow} \Omega \xi^{\dagger} \Omega
$$

as well as invariant under global $\mathrm{SU}(5)$ transformations,

$$
\Sigma=\xi^{2} \Sigma_{0} \longrightarrow V \Sigma V^{T} \Rightarrow \xi \longrightarrow V \xi U^{\dagger} \equiv U \xi \Sigma_{0} V^{T} \Sigma_{0},
$$


where $V$ is the global SU(5) transformation while $U$ takes values in the $\mathrm{SO}(5)$ Lie algebra and is a function of $V$ and $\Pi$. We note that the gauge singlet $\chi_{R}$ is T-even and is assumed to be heavy since it plays no role in the following. ${ }^{7}$

The remaining leptons become massive through two other different Yukawa Lagrangians. The SM combination $l_{L}=\frac{l_{1 L}-l_{2 L}}{\sqrt{2}}$ in eqs. (2.13)-(2.15) will obtain a mass through the following Yukawa couplings $[13,19]$

$$
\mathcal{L}_{Y}=\frac{i \lambda}{2 \sqrt{2}} f \epsilon^{x y z} \epsilon^{r s}\left[\left(\overline{\Psi_{2}^{\chi}}\right)_{x}(\Sigma)_{r y}(\Sigma)_{s z}+\left(\overline{\Psi_{1}^{\tilde{\chi}}} \Sigma_{0} \Omega\right)_{x}(\widetilde{\Sigma})_{r y}(\widetilde{\Sigma})_{s z}\right] \ell_{R}+\text { h.c. }
$$

where summation over $x, y, z=3,4,5$ and $r, s=1,2$ is understood.

Although all SU(5) indices are contracted and the SM right-handed leptons are assumed to be $\mathrm{SU}(5)$ singlets, this Lagrangian is not invariant under the $\mathrm{SU}(5)$ global symmetry. In addition to the breaking due to $\Sigma_{0}$ and the incomplete $\Psi_{1,2}$ multiplets, the $\Psi_{1,2}$ non-zero entries do not have the proper quantum numbers to construct the SM terms. Indeed, in order to enforce gauge invariance under the SM, extra U(1) charges outside the $\mathrm{SU}(5)$ are needed since $\ell_{R}$ is required to have hypercharge -1 . Then, the term in brackets in eq. (2.21) must have hypercharge 1, but being an $\mathrm{SU}(5)$ singlet this $\mathrm{U}(1)$ charge must lie outside the $\mathrm{SU}(5)$ as well.

Following refs. $[13,19,29]$ we compose two other incomplete SU(5) multiplets in fundamental representations $\underline{5}$ and $\underline{5}^{*}$, respectively:

$$
\Psi_{1}^{\tilde{\chi}}=\left(\begin{array}{c}
\tilde{\chi} l_{1 L} \\
0 \\
0
\end{array}\right), \quad \Psi_{2}^{\chi}=\left(\begin{array}{c}
0 \\
0 \\
\chi l_{2 L}
\end{array}\right),
$$

where $\chi$ is a scalar with the proper charges to endow $\chi l_{2 L}$ with the charges corresponding to the last two components of $\underline{5}^{*}$. More precisely, we will only require that it transforms properly under its gauged subgroup. The charge assignments fulfilling this requirement are gathered in table 1 [29]. Thus, the introduction of $\chi$ allows us to change the sign of the gauged $\mathrm{U}(1)$ charges in $\mathrm{SU}(5)$ for $l_{2 L}$ while also giving the correct hypercharge to $\chi l_{2 L}$. The action under T-parity is then defined as

$$
\Psi_{1}^{\tilde{\chi}} \stackrel{\mathrm{T}}{\longleftrightarrow} \Omega \Sigma_{0} \Psi_{2}^{\chi}
$$

An explicit realization of this extra scalar factor is obtained identifying $\chi$ with $\left(\Sigma_{33}^{\dagger}\right)^{\frac{1}{4}}$, and $\tilde{\chi}$ with $\left(\Sigma_{33}\right)^{-\frac{1}{4}}$, which have the correct $Y_{1,2}$ charges, $\left(Y_{1}^{\chi}, Y_{2}^{\chi}\right)=\left(-\frac{1}{10}, \frac{1}{10}\right)$ and $\left(Y_{1}^{\chi}, Y_{2}^{\tilde{\chi}}\right)=$ $\left(\frac{1}{10},-\frac{1}{10}\right)$, and T-transformation properties [19]. ${ }^{8}$

Turning back to the partner lepton doublets $\tilde{\psi}_{R}$ in eq. (2.16) they are T-odd, as desired, but must be heavy enough to agree with EWPD. Since they do not receive a mass from

\footnotetext{
${ }^{7}$ If we had defined the $\mathrm{T}$ action on the fermions $\Psi_{1} \stackrel{\mathrm{T}}{\longleftrightarrow}-\Sigma_{0} \Psi_{2}, \Psi_{R} \stackrel{\mathrm{T}}{\longrightarrow}-\Psi_{R}$ and the Yukawa Lagrangian with $\Omega$ 's, $\mathcal{L}_{Y_{H}}=-\kappa f\left(\bar{\Psi}_{2} \xi+\bar{\Psi}_{1} \Sigma_{0} \Omega \xi^{\dagger} \Omega\right) \Psi_{R}+$ h.c., all new fermions would be T-odd and the new Lagrangian invariant under the new T-parity [12], but not under the full global symmetry because $\Omega$ does not commute with $\mathrm{SU}(5)$ nor with $\mathrm{SO}(5)$, though it does commute with the gauge group. Regardless, the explicit couplings entering in our calculation are the same in both cases.

${ }^{8}$ This particular realization will not play an essential role in $h \rightarrow \tau \mu$ at the order to which we work.
} 


\begin{tabular}{|c|cccc|}
\hline & $Y_{1}^{\prime}$ & $Y_{2}^{\prime}$ & $Y_{1}^{\prime \prime}$ & $Y_{2}^{\prime \prime}$ \\
\hline$\chi$ & $\frac{2}{5}$ & $\frac{3}{5}$ & $-\frac{1}{2}$ & $-\frac{1}{2}$ \\
$\tilde{\chi}$ & $\frac{3}{5}$ & $\frac{2}{5}$ & $-\frac{1}{2}$ & $-\frac{1}{2}$ \\
$l_{2 L}$ & $-\frac{1}{5}$ & $-\frac{3}{10}$ & 0 & 0 \\
$l_{1 L}$ & $-\frac{3}{10}$ & $-\frac{1}{5}$ & 0 & 0 \\
\hline$\chi l_{2 L}$ & $\frac{1}{5}$ & $\frac{3}{10}$ & $-\frac{1}{2}$ & $-\frac{1}{2}$ \\
$\tilde{\chi} l_{1 L}$ & $\frac{3}{10}$ & $\frac{1}{5}$ & $-\frac{1}{2}$ & $-\frac{1}{2}$ \\
\hline
\end{tabular}

Table 1. Charge assignment under $\mathrm{U}(1)_{1}^{\prime} \times \mathrm{U}(1)_{2}^{\prime} \times \mathrm{U}(1)_{1}^{\prime \prime} \times \mathrm{U}(1)_{2}^{\prime \prime}$, where the first two single prime factors are the abelian subgroups inside $\mathrm{SU}(5)$ while the double primed abelian groups live outside. The gauged $\mathrm{U}(1)_{1} \times \mathrm{U}(1)_{2}$ correspond to the sums $Y_{b}=Y_{b}^{\prime}+Y_{b}^{\prime \prime}, b=1,2$ where the total sum $Y=Y_{1}+Y_{2}$ gives the hypercharge.

the Yukawa couplings in eq. (2.18) as the mirror leptons do, they remain massless as long as no other left-handed doublet is introduced to allow for the generation of a vector-like mass. The corresponding mechanism giving them a mass provides a new source of LFV if misaligned with the Yukawa couplings in eqs. (2.18) and (2.21), making the discussion of their origin essential. Of course, as already emphasized and to be shown explicitly below, these partner leptons are also required to obtain a finite amplitude for $h \rightarrow \tau \mu$.

A simple solution for giving $\tilde{\psi}_{R}$ a mass is to write an explicit mass term with an incomplete $\mathrm{SO}(5)$ defining representation $\Psi_{L}=\left(\tilde{\psi}_{L}, 0,0\right)^{T}$ which includes a left-handed counterpart $\tilde{\psi}_{L}=-i \sigma^{2}\left(\tilde{l}_{R}\right)^{c}$ with which to form a vector like mass term $[11,12]$. There are other ways to generate a mass, but regardless of which mechanism is assumed it necessarily, but softly, breaks the $\mathrm{SO}(5)$ to give a large mass to $\tilde{\psi}_{R}$ (and/or $\chi_{R}$ ) alone in the $\Psi_{R}$ multiplet. ${ }^{9}$ This enters in corrections to the Higgs self-energy for which the $\tilde{\psi}_{R}$ mass serves as a finite cutoff at two loops $[11,12]$. Thus these masses can not be taken arbitrarily large without reintroducing a fine tuning into the Higgs mass. As we show below, this soft breaking of $\mathrm{SO}(5)$ also manifests itself at one loop and $\mathcal{O}\left(v^{2} / f^{2}\right)$ in $h \rightarrow \tau \mu$ as a logarithmic non-decoupling behavior when the partner lepton masses are taken large. In our analysis we will simply parameterize the partner lepton masses as follows,

$$
\mathcal{L}_{M}=-M^{T} \overline{\tilde{\psi}_{L}} \tilde{\psi}_{R}+\text { h.c. }=-M \tilde{\tilde{l}}_{L} \tilde{l}_{R}+\text { h.c. },
$$

where $\tilde{\psi}_{R}=-i \sigma^{2}\left(\tilde{l}_{L}\right)^{c}$ and $\tilde{l}_{L}=\left(\tilde{\nu}_{L} \tilde{\ell}_{L}\right)^{T}$ (with $T$ meaning transpose).

\footnotetext{
${ }^{9}$ We could instead include the left-handed counterpart of $\tilde{\psi}_{R}$ in an SO(5) spinor (pseudo-real) representation $\underline{4}$, giving them a mass through a Yukawa coupling with new scalars also transforming as a $\underline{4}$, for $\underline{5} \subset \underline{4} \times \underline{4}$. In this way $\chi_{R}$ in $\Psi_{R}$ would also receive a mass when the SM neutral singlet within the scalar spinor representation gets a vev. In contrast the SM neutral singlet lepton in the left-handed fermion multiplet $\underline{4}$ does not receive any mass because $\underline{5}$ is in the antisymmetric product of $\underline{4} \times \underline{4}$. (As already emphasized, the embedding of $\mathrm{SO}(5) \subset \mathrm{SU}(5)$ implied by the breaking along $\Sigma_{0}$ in eq. (2.1) reduces the fundamental representation of $\mathrm{SU}(5)$ to the defining (real) representation of $\mathrm{SO}(5), \underline{5}=\underline{5}$; and no pseudo-real representation is generated by tensor product of real ones. Hence, the vev along the spinor representation of $\mathrm{SO}(5)$ not only breaks this global symmetry group but its eventual embedding in an SU(5) representation would be different to the assumed one in the non-linear realization of the LHT.)
} 
Although it does not have any consequence in our calculation, we comment that the Yukawa-type Lagrangian $\mathcal{L}_{Y_{H}}$ fixes the transformation properties of the heavy fermions including their gauge couplings. In particular the non-linear couplings of the right-handed heavy fermions $[11,13]$ are fixed to be,

$$
\begin{aligned}
\mathcal{L}_{F}= & i \bar{\Psi}_{1} \gamma^{\mu} D_{\mu}^{*} \Psi_{1}+i \bar{\Psi}_{2} \gamma^{\mu} D_{\mu} \Psi_{2} \\
& +i \overline{\Psi_{R}} \gamma^{\mu}\left(\partial_{\mu}+\frac{1}{2} \xi^{\dagger}\left(D_{\mu} \xi\right)+\frac{1}{2} \xi\left(\Sigma_{0} D_{\mu}^{*} \Sigma_{0} \xi^{\dagger}\right)\right) \Psi_{R} \\
& +\Psi_{R} \rightarrow \Psi_{L}
\end{aligned}
$$

with the covariant derivative defined as

$$
D_{\mu}=\partial_{\mu}-\sqrt{2} i g\left(W_{1 \mu}^{a} Q_{1}^{a}+W_{2 \mu}^{a} Q_{2}^{a}\right)+\sqrt{2} i g^{\prime}\left(Y_{1} B_{1 \mu}+Y_{2} B_{2 \mu}\right) .
$$

This Lagrangian includes the proper $\mathcal{O}\left(v^{2} / f^{2}\right)$ couplings to Goldstone fields that render the one-loop lepton flavor changing amplitudes mediated by gauge bosons ultraviolet finite [25, 26]. Finally, as discussed above, in order to assign the proper SM hypercharge $Y=-1$ to the charged right-handed leptons $\ell_{R}$, one can enlarge the global SU(5) with two extra U(1) groups for which we can write down the corresponding gauge and T-invariant Lagrangian

$$
\mathcal{L}_{F}^{\prime}=i \bar{\ell}_{R} \gamma^{\mu}\left(\partial_{\mu}+i g^{\prime} Y B_{\mu}\right) \ell_{R}
$$

These are all the necessary Lagrangian terms for the lepton sector and the type of process we are interested in, up to family indices that we shall introduce in the following. In order to perform the calculation in the mass eigenstate basis we have to diagonalize the full Lagrangian

$$
\mathcal{L}=\mathcal{L}_{G}+\mathcal{L}_{S}+\mathcal{L}_{Y_{H}}+\mathcal{L}_{Y}+\mathcal{L}_{M}+\mathcal{L}_{F}+\mathcal{L}_{F}^{\prime}
$$

and re-express it in the mass eigenstate basis. The corresponding masses and eigenfields up to $\mathcal{O}\left(v^{2} / f^{2}\right)$ and the relevant Feynman rules are collected below and are obtained by expanding $\mathcal{L}$ to the required order.

\subsection{Mass eigenfields and Feynman rules}

An important technical part of this paper is to prove unambiguously the finiteness of the process under study ( $h \rightarrow \bar{\ell} \ell^{\prime}$ ), and the need to include the full set of T-odd scalars and fermions. We will discuss the details of the calculation in next section, but first we collect here all the required Feynman rules and define the mass eigenstates.

\subsubsection{Mass eigenfields}

Gauge fields. After EWSB the SM gauge boson mass eigenstates (see eq. (2.7)), which are the T-even, are obtained by diagonalizing $\mathcal{L}_{S}$ in eq. (2.11):

$$
W^{ \pm}=\frac{1}{\sqrt{2}}\left(W^{1} \mp i W^{2}\right), \quad\left(\begin{array}{c}
Z \\
A
\end{array}\right)=\left(\begin{array}{cc}
c_{W} & s_{W} \\
-s_{W} & c_{W}
\end{array}\right)\left(\begin{array}{c}
W^{3} \\
B
\end{array}\right),
$$


with

$$
W^{a}=\frac{W_{1}^{a}+W_{2}^{a}}{\sqrt{2}}, \quad B=\frac{B_{1}+B_{2}}{\sqrt{2}}
$$

whereas the T-odd mass eigenstates, expanding the heavy sector up to order $v^{2} / f^{2}$, are (see eq. (2.8)):

$$
W_{H}^{ \pm}=\frac{1}{\sqrt{2}}\left(W_{H}^{1} \mp i W_{H}^{2}\right), \quad\left(\begin{array}{c}
Z_{H} \\
A_{H}
\end{array}\right)=\left(\begin{array}{cc}
1 & -x_{H} \frac{v^{2}}{f^{2}} \\
x_{H} \frac{v^{2}}{f^{2}} & 1
\end{array}\right)\left(\begin{array}{c}
W_{H}^{3} \\
B_{H}
\end{array}\right),
$$

with

$$
W_{H}^{a}=\frac{W_{1}^{a}-W_{2}^{a}}{\sqrt{2}}, \quad B_{H}=\frac{B_{1}-B_{2}}{\sqrt{2}}, \quad x_{H}=\frac{5 g g^{\prime}}{4\left(5 g^{2}-g^{\prime 2}\right)} .
$$

Their masses to order $v^{2} / f^{2}$ are (with $e$ the electric charge, $s_{W}\left(c_{W}\right)=\sin \theta_{W}\left(\cos \theta_{W}\right)$, with $\theta_{W}$ the electroweak mixing angle, and $v \simeq 246 \mathrm{GeV}$ )

$$
\begin{array}{rlrl}
M_{W} & =\frac{g v}{2}\left(1-\frac{v^{2}}{12 f^{2}}\right), & M_{Z} & =M_{W} / c_{W}, \quad e=g s_{W}=g^{\prime} c_{W}, \\
M_{W_{H}}=M_{Z_{H}}=g f\left(1-\frac{v^{2}}{8 f^{2}}\right), & M_{A_{H}}=\frac{g^{\prime} f}{\sqrt{5}}\left(1-\frac{5 v^{2}}{8 f^{2}}\right) . &
\end{array}
$$

Scalar fields. The scalar fields must be also rotated into the mass basis [14]:

$$
\begin{aligned}
\pi^{0} & \rightarrow \pi^{0}\left(1+\frac{v^{2}}{12 f^{2}}\right), \\
\pi^{ \pm} & \rightarrow \pi^{ \pm}\left(1+\frac{v^{2}}{12 f^{2}}\right), \\
h & \rightarrow h, \\
\Phi^{0} & \rightarrow \Phi^{0}\left(1+\frac{v^{2}}{12 f^{2}}\right), \\
\Phi^{P} & \rightarrow \Phi^{P}+\left(\sqrt{10} \eta-\sqrt{2} \omega^{0}+\Phi^{P}\right) \frac{v^{2}}{12 f^{2}}, \\
\Phi^{ \pm} & \rightarrow \Phi^{ \pm}\left(1+\frac{v^{2}}{24 f^{2}}\right) \pm i \omega^{ \pm} \frac{v^{2}}{12 f^{2}}, \\
\Phi^{++} & \rightarrow \Phi^{++}, \\
\eta & \rightarrow \eta+\frac{5 g^{\prime} \eta-4 \sqrt{5}\left[g^{\prime}\left(\omega^{0}+\sqrt{2} \Phi^{P}\right)-6 g x_{H} \omega^{0}\right]}{24 g^{\prime}} \frac{v^{2}}{f^{2}}, \\
\omega^{0} & \rightarrow \omega^{0}+\frac{5 g\left(\omega^{0}+4 \sqrt{2} \Phi^{P}\right)-4 \sqrt{5} \eta\left(5 g+6 g^{\prime} x_{H}\right)}{120 g} \frac{v^{2}}{f^{2}}, \\
\omega^{ \pm} & \rightarrow \omega^{ \pm}\left(1+\frac{v^{2}}{24 f^{2}}\right) \pm i \Phi^{ \pm} \frac{v^{2}}{6 f^{2}} .
\end{aligned}
$$


The fields $\eta, \omega^{0}$ and $\omega^{ \pm}$are the Goldstone bosons of the gauge group $\mathrm{SU}(2)_{1} \times \mathrm{SU}(2)_{2} \times$ $\mathrm{U}(1)_{1} \times \mathrm{U}(1)_{2}$ breaking into the SM. They are eaten by the heavy gauge bosons $A_{H}, Z_{H}$ and $W_{H}^{ \pm}$, respectively, while $\pi^{0}$ and $\pi^{ \pm}$are the Goldstone bosons of the SM gauge group. The physical pseudo-Goldstone bosons include the Higgs boson with mass $M_{h} \simeq 125 \mathrm{GeV}$ [41], and the scalar triplet of hypercharge 1 , with a mass $M_{\Phi}$ which we assume to be a free parameter of order $f$ [17], though both masses are a priori calculable since they can be obtained in the LHT through a Coleman-Weinberg mechanism [42].

Fermionic fields. Fermion masses and mass eigenvectors are obtained from the diagonalization of the $3 \times 3$ matrices $\kappa, \lambda$ and $M$ in $\mathcal{L}_{Y_{H}}, \mathcal{L}_{Y}$ and $\mathcal{L}_{M}$, respectively. For each of the three SM left-handed lepton doublets there is an extra vector-like doublet,

$$
l_{r L}=\left(\begin{array}{c}
\nu_{r L} \\
\ell_{r L}
\end{array}\right), \quad r=1,2, \quad l_{H R}=\left(\begin{array}{c}
\nu_{H R} \\
\ell_{H R}
\end{array}\right),
$$

with (see eqs. (2.13)-(2.17))

$$
l_{L}=\frac{l_{1 L}-l_{2 L}}{\sqrt{2}}, \quad l_{H L}=\frac{l_{1 L}+l_{2 L}}{\sqrt{2}}, \quad l=\nu, \ell,
$$

where we have omitted flavor indices. The fields $\nu_{L}, \ell_{L}$ are the SM (T-even) left-handed leptons and $\nu_{H L}, \ell_{H L}\left(\nu_{H R}, \ell_{H R}\right)$ are T-odd left (right) handed leptons with masses $\mathcal{O}(f)$. In addition to three SM right-handed charged leptons $\ell_{R}$, which are assumed to be singlets under the non-abelian symmetries, there is a second heavy vector-like doublet per family, $\tilde{l}_{L}=\left(\tilde{\nu}_{L} \tilde{\ell}_{L}\right)^{T}$ and its Dirac partner $\tilde{l}_{R}=\left(\tilde{\nu}_{R} \tilde{\ell}_{R}\right)^{T}$. These come from the partner leptons $\tilde{\psi}_{R}$ needed to complete the $\mathrm{SO}(5)$ multiplet $\Psi_{R}$ in eqs. (2.16) and its partner $\tilde{\psi}_{L}$ in eq. (2.24).

Let us now introduce flavor indices and masses and mass eigenleptons, which we shall denote as the current eigenfermions but with family indices. Since T-parity is exact, the T-even SM charged leptons $\ell$ do not mix with the heavy T-odd leptons. Thus the SM mass eigenstates result from the diagonalization of the $3 \times 3$ matrix $\lambda$ in eq. (2.21)

$$
\frac{\lambda_{i j}}{\sqrt{2}} v=\left(V_{L}^{\ell}\right)_{i k} m_{\ell_{k}}\left(V_{R}^{\ell \dagger}\right)_{k j}
$$

where $i, j, k=1,2,3, V_{L, R}^{\ell}$ are $3 \times 3$ unitary matrices and $m_{\ell_{1,2,3}}=m_{e, \mu, \tau}$ (up to loop corrections $\left.\mathcal{O}\left(v^{2} / f^{2}\right)\right)$. The charged T-odd lepton masses result from diagonalizing a $6 \times 6$ matrix, which is block diagonal (see eqs. (2.18) and (2.24)),

$$
\left(\begin{array}{cc}
\kappa_{i j} f \sqrt{2} & 0 \\
0 & M_{i^{\prime} j^{\prime}}
\end{array}\right)=\left(\begin{array}{cc}
\left(V_{L}^{H}\right)_{i k} & 0 \\
0 & \left(\tilde{V}_{L}\right)_{i^{\prime} k^{\prime}}
\end{array}\right)\left(\begin{array}{cc}
m_{\ell_{H k}} & 0 \\
0 & m_{\tilde{\ell}_{k^{\prime}}}
\end{array}\right)\left(\begin{array}{cc}
\left(V_{R}^{H \dagger}\right)_{k j} & 0 \\
0 & \left(\tilde{V}_{R}^{\dagger}\right)_{k^{\prime} j^{\prime}}
\end{array}\right)
$$

where $i, j, k, i^{\prime}, j^{\prime}, k^{\prime}=1,2,3$ and $m_{\ell_{H 1,2,3}, \tilde{\ell}_{1,2,3}}$ are the mass eigenvalues, and $V_{L, R}^{H}, \tilde{V}_{L, R}$ are $3 \times 3$ unitary matrices. ${ }^{10}$ The heavy (T-odd) charged and neutral leptons have the same

\footnotetext{
${ }^{10}$ The Yukawa Lagrangian in eq. (2.18) does not mix T-odd charged leptons without and with tilde because they are embedded with opposite (hyper)charges in $\Psi_{R}$.
} 
mass and diagonalization matrices at leading order. At next order the $3 \times 3$ top-left block gets a correction $\sqrt{2} \kappa_{i j} f \rightarrow \sqrt{2} \kappa_{i j} f\left(1-\frac{v^{2}}{8 f^{2}}\right)$ and hence,

$$
m_{\nu_{H k}}=m_{\ell_{H k}}\left(1-\frac{v^{2}}{8 f^{2}}\right)
$$

There is also a correction of this order combining $\bar{\nu}_{H L}$ and $\left(\tilde{\nu}_{L}\right)^{c}$ in which case a diagonalization of the corresponding $12 \times 12$ mass matrix for the heavy neutrinos is needed. This gives corrections to heavy neutrino masses which are $\mathcal{O}\left(v^{4} / f^{4}\right)$ and mixings among heavy neutrinos $\mathcal{O}\left(v^{2} / f^{2}\right)$. This translates into higher order $\mathcal{O}\left(v^{4} / f^{4}\right)$ corrections to the one-loop Higgs decay under study and is therefore neglected.

The misalignment between the mass matrices of the T-even (SM) and T-odd (heavy) leptons is a source of flavor mixing. We can work without loss of generality in the mass eigenstate basis diagonalizing the SM Yukawa couplings in $\mathcal{L}_{Y}$. We can then write the mixing matrices parameterizing the misalignment between the different leptonic sectors as

$$
V \equiv V_{L}^{H \dagger} V_{L}^{\ell}, \quad W \equiv \tilde{V}_{L}^{T} V_{L}^{H}
$$

These will enter explicitly into the Feynman rules and amplitudes we study below. As we will see, the new source of LFV we emphasize here enters through the mixing matrix $W$.

\subsubsection{Feynman rules}

In order to calculate the Higgs decay into two different charged leptons at one loop, we need to complete the Feynman rules for the LHT worked out previously in the literature. ${ }^{11}$ In particular, we have to work out the terms of $\mathcal{L}_{S}+\mathcal{L}_{Y_{H}}+\mathcal{L}_{Y}$ involving a Higgs up to order $v^{2} / f^{2}$. We just present the Feynman rules in the mass eigenstate basis which are necessary for the calculation of the LFV processes discussed in this work. They are given in tables $2-5$ in terms of generic couplings for the following general vertices involving scalars (S), fermions (F) and/or gauge bosons (V):

$$
\begin{aligned}
{[\mathrm{SFF}] } & =i\left(c_{L} P_{L}+c_{R} P_{R}\right), \\
{\left[\mathrm{SV}_{\mu} \mathrm{V}_{\nu}\right] } & =i K g^{\mu \nu} \\
{\left[\mathrm{V}_{\mu} \mathrm{FF}\right] } & =i \gamma^{\mu}\left(g_{L} P_{L}+g_{R} P_{R}\right), \\
{[\mathrm{SSFF}] } & =i\left(f_{L} P_{L}+f_{R} P_{R}\right), \\
{\left[\mathrm{S}\left(p_{1}\right) \mathrm{S}\left(p_{2}\right) \mathrm{V}_{\mu}\right] } & =i G\left(p_{1}-p_{2}\right)^{\mu}, \\
{\left[\mathrm{SS}\left(p_{1}\right) \mathrm{S}\left(p_{2}\right)\right] } & =i J\left(p_{1}^{2}+p_{2}^{2}+4 p_{1} \cdot p_{2}\right),
\end{aligned}
$$

where all momenta are assumed incoming. The conjugate vertices are obtained replacing:

$$
c_{L, R} \leftrightarrow c_{R, L}^{*}, \quad K \leftrightarrow K^{*}, g_{L, R} \leftrightarrow g_{L, R}^{*}, \quad f_{L, R} \leftrightarrow f_{R, L}^{*}, \quad G \leftrightarrow G^{*}, J \leftrightarrow J^{*} .
$$

\footnotetext{
${ }^{11}$ In particular, in ref. [26] the Feynman rules in appendix B.2 for one scalar and two right-handed leptons, $c_{R}$ in SFF, have a typo. The flavor subscript for $m_{\ell_{i}}$ should be $j$, and not $i$. The sign conventions are chosen to be compatible with those employed for the SM in ref. [40], which coincide with those in ref. [39] up to a sign in the definition of the abelian gauge couplings in the covariant derivative in eq. (2.12).
} 


\begin{tabular}{|c|c|c|}
\hline$[\mathrm{SFF}]$ & $c_{L}$ & $c_{R}$ \\
\hline$h \overline{\ell_{i}} \ell_{j}$ & $-\delta_{i j} \frac{m_{\ell_{j}}}{v}\left(1-\frac{v^{2}}{6 f^{2}}\right)$ & $-\delta_{i j} \frac{m_{\ell_{j}}}{v}\left(1-\frac{v^{2}}{6 f^{2}}\right)$ \\
$h \overline{\nu_{H i}} \nu_{H j}$ & $\frac{m_{\ell_{H i}}}{v} \delta_{i j} \frac{v^{2}}{4 f^{2}}$ & $\frac{m_{\ell_{H i}}}{v} \delta_{i j} \frac{v^{2}}{4 f^{2}}$ \\
$h \overline{\ell_{H i}} \ell_{H j}$ & 0 & 0 \\
$h \overline{\nu_{H i}} \tilde{\nu}_{j}^{c}$ & $\frac{m_{\ell_{H i}}}{v} W_{i j}^{\dagger} \frac{v^{2}}{4 f^{2}}$ & $\frac{m_{\ell_{H i}}}{v} W_{i j}^{\dagger} \frac{v^{2}}{4 f^{2}}$ \\
$\Phi^{0} \overline{\ell_{H i}} \ell_{j}$ & 0 & $V_{i j} \frac{m_{\ell_{j}}}{\sqrt{2} f}\left(1+\frac{v^{2}}{4 f^{2}}\right)$ \\
$\Phi^{P} \overline{\ell_{H i}} \ell_{j}$ & 0 & $i V_{i j} \frac{m_{\ell_{j}}}{\sqrt{2} f}\left(1+\frac{v^{2}}{4 f^{2}}\right)$ \\
$\Phi^{+} \overline{\nu_{H i}} \ell_{j}$ & $\frac{m_{\ell_{H i}}}{\sqrt{2} f} V_{i j} \frac{v^{2}}{8 f^{2}}$ & $V_{i j} \frac{m_{\ell_{j}}}{\sqrt{2} f}\left(1-\frac{v^{2}}{8 f^{2}}\right)$ \\
$\Phi^{+} \overline{\tilde{\nu}_{i}^{c}} \ell_{j}$ & $W_{i k} \frac{m_{\ell_{H k}}}{\sqrt{2} f} V_{k j}$ & 0 \\
$\Phi^{++} \overline{\tilde{\ell}_{i}^{c}} \ell_{j}$ & $-W_{i k} \frac{m_{\ell_{H k}}}{f} V_{k j}$ & 0 \\
$\eta \overline{\ell_{H i}} \ell_{j}$ & $i \frac{m_{\ell_{H i}}}{2 \sqrt{5} f} V_{i j}\left(1-x_{H} t_{W} \frac{v^{2}}{f^{2}}\right)$ & $-i V_{i j} \frac{m_{\ell_{j}}}{2 \sqrt{5} f}\left[1+\left(\frac{11}{24}-x_{H} t_{W}\right) \frac{v^{2}}{f^{2}}\right]$ \\
$\omega^{0} \overline{\ell_{H i}} \ell_{j}$ & $i \frac{m_{\ell_{H i}}}{2 f} V_{i j}\left(1+\frac{x_{H}}{t_{W}} \frac{v^{2}}{f^{2}}\right)$ & $-i V_{i j} \frac{m_{\ell_{j}}}{2 f}\left[1-\left(\frac{1}{8}-\frac{x_{H}}{t_{W}}\right) \frac{v^{2}}{f^{2}}\right]$ \\
$\omega^{+} \overline{\nu_{H i}} \ell_{j}$ & $-i \frac{m_{\ell_{H i}}}{\sqrt{2} f} V_{i j}$ & $i V_{i j} \frac{m_{\ell_{j}}}{\sqrt{2} f}\left(1+\frac{v^{2}}{8 f^{2}}\right)$ \\
$\omega^{+} \overline{\tilde{\nu}_{i}^{c}} \ell_{j}$ & $i W_{i k} \frac{m_{\ell_{H k}}}{\sqrt{2} f} V_{k j} \frac{v^{2}}{8 f^{2}}$ & 0 \\
\hline
\end{tabular}

Table 2. Scalar-Fermion-Fermion couplings at $\mathcal{O}\left(v^{2} / f^{2}\right)$. The corresponding couplings involving the $\Psi_{R i}$ neutral singlet, $\chi_{R i}$, vanish. We use $t_{W}=\tan \theta_{W}=\frac{s_{W}}{c_{W}}$. (The coupling $\Phi^{++} \overline{\tilde{\ell}_{i}^{c}} \ell_{j}$ does not enter in the calculation of $h \rightarrow \bar{\ell} \ell^{\prime}$, but it does in $Z, \gamma \rightarrow \bar{\ell} \ell^{\prime}$ in order to cancel other contributions.)

\begin{tabular}{|c|c|}
\hline$\left[\mathrm{SV}_{\mu} \mathrm{V}_{\nu}\right]$ & $K$ \\
\hline$h W_{H}^{+} W_{H}^{-}$ & $-g^{2} \frac{v}{2}$ \\
$h Z_{H} Z_{H}$ & $-g^{2} \frac{v}{4}$ \\
$h A_{H} A_{H}$ & $-g^{\prime 2 \frac{v}{4}}$ \\
$h Z_{H} A_{H}$ & $-g g^{\prime} \frac{v}{4}$ \\
\hline
\end{tabular}

\begin{tabular}{|c|c|}
\hline$\left[\mathrm{V}_{\mu} \mathrm{FF}\right]$ & $g_{L}$ \\
\hline$W_{H}^{+} \overline{\nu_{H i}} \ell_{j}$ & $\frac{g}{\sqrt{2}} V_{i j}$ \\
$Z_{H} \overline{\ell_{H i}} \ell_{j}$ & $-\left(\frac{g}{2}+\frac{g^{\prime}}{10} x_{H} \frac{v^{2}}{f^{2}}\right) V_{i j}$ \\
$A_{H} \overline{\ell_{H i}} \ell_{j}$ & $\left(\frac{g^{\prime}}{10}-\frac{g}{2} x_{H} \frac{v^{2}}{f^{2}}\right) V_{i j}$ \\
\hline
\end{tabular}

Table 3. Scalar-Vector-Vector and Vector-Fermion-Fermion couplings at $\mathcal{O}\left(v^{2} / f^{2}\right)$. The righthanded Vector-Fermion-Fermion couplings $g_{R}$ vanish.

\section{Higgs coupling to a pair of different charged leptons at one loop in the LHT}

The global symmetries of the LHT prevent tree level LFV Higgs decays, but they are generated at one loop via the T-odd particles. Since they are forbidden at tree level, the one loop decays are finite and a prediction of the LHT as we explicitly show below. To 


\begin{tabular}{|c|c|c|}
\hline$[\mathrm{SSFF}]$ & $f_{L}$ & $f_{R}$ \\
\hline$h \Phi^{0} \overline{\ell_{H i}} \ell_{j}$ & 0 & $V_{i j} \frac{m_{\ell_{j}}}{\sqrt{2} v f}\left(1+\frac{5 v^{2}}{12 f^{2}}\right)$ \\
$h \Phi^{P} \overline{\ell_{H i}} \ell_{j}$ & 0 & $i V_{i j} \frac{m_{\ell_{j}}}{\sqrt{2} v f}\left(1+\frac{5 v^{2}}{12 f^{2}}\right)$ \\
$h \Phi^{+} \overline{\nu_{H i}} \ell_{j}$ & $-\frac{m_{\ell_{H i}}}{\sqrt{2} v f} V_{i j} \frac{v^{2}}{12 f^{2}}$ & $V_{i j} \frac{m_{\ell_{j}}}{\sqrt{2} v f}\left(1-\frac{7 v^{2}}{24 f^{2}}\right)$ \\
$h \Phi^{+} \overline{\tilde{\nu}_{i}^{c}} \ell_{j}$ & $-W_{i k} \frac{m_{\ell_{H k}}}{\sqrt{2} v f} V_{k j} \frac{v^{2}}{12 f^{2}}$ & 0 \\
$h \eta \overline{\ell_{H i}} \ell_{j}$ & 0 & $-i V_{i j} \frac{m_{\ell_{j}}}{2 \sqrt{5} v f}\left[1+\left(\frac{47}{24}-x_{H} \frac{s_{W}}{c_{W}}\right) \frac{v^{2}}{f^{2}}\right]$ \\
$h \omega^{0} \overline{\ell_{H i}} \ell_{j}$ & 0 & $-i V_{i j} \frac{m_{\ell_{j}}}{2 v f}\left[1-\left(\frac{5}{8}-x_{H} \frac{c_{W}}{s_{W}}\right) \frac{v^{2}}{f^{2}}\right]$ \\
$h \omega^{+} \overline{\overline{\nu_{H i}}} \ell_{j}$ & $i \frac{m_{\ell_{H i}}}{\sqrt{2} v f} V_{i j} \frac{v^{2}}{12 f^{2}}$ & $i V_{i j} \frac{m_{\ell_{j}}}{\sqrt{2} v f}\left(1-\frac{v^{2}}{24 f^{2}}\right)$ \\
$h \omega^{+} \overline{\tilde{\nu}_{i}^{c}} \ell_{j}$ & $i W_{i k} \frac{m_{\ell_{H k}}}{\sqrt{2} v f} V_{k j} \frac{v^{2}}{12 f^{2}}$ & 0 \\
\hline
\end{tabular}

Table 4. Scalar-Scalar-Fermion-Fermion couplings at $\mathcal{O}\left(v^{2} / f^{2}\right)$. The corresponding couplings involving the $\Psi_{R i}$ neutral singlet, $\chi_{R i}$, do not vanish, but at the order in which we work they do not contribute to the processes we are interested in. The coupling $h \phi^{++} \overline{\tilde{\ell}_{i}^{c}} \ell_{j}$ does vanish.

\begin{tabular}{|c|c|}
\hline$\left[\mathrm{S}\left(p_{1}\right) \mathrm{S}\left(p_{2}\right) \mathrm{V}_{\mu}\right]$ & $G$ \\
\hline$h \Phi^{+} W_{H}^{-}$ & $g \frac{v}{4 f}$ \\
$h \eta A_{H}$ & $i \sqrt{5} g^{\prime} \frac{v}{4 f}$ \\
$h \omega^{0} Z_{H}$ & $-i g \frac{v}{4 f}$ \\
$h \omega^{+} W_{H}^{-}$ & $-i g \frac{v}{4 f}$ \\
\hline
\end{tabular}

\begin{tabular}{|c|c|}
\hline$\left[\mathrm{SS}\left(p_{1}\right) \mathrm{S}\left(p_{2}\right)\right]$ & $J$ \\
\hline$h \Phi^{0} \Phi^{0}$ & $\frac{v}{12 f^{2}}$ \\
$h \Phi^{P} \Phi^{P}$ & $\frac{v}{12 f^{2}}$ \\
$h \Phi^{P} \eta$ & $-\sqrt{\frac{5}{2}} \frac{v}{24 f^{2}}$ \\
$h \Phi^{P} \omega^{0}$ & $\frac{1}{\sqrt{2}} \frac{v}{24 f^{2}}$ \\
$h \Phi^{+} \Phi^{-}$ & $\frac{v}{12 f^{2}}$ \\
$h \Phi^{+} \omega^{-}$ & $i \frac{v}{12 f^{2}}$ \\
$h \eta \eta$ & $\frac{5 v}{12 f^{2}}$ \\
$h \eta \omega^{0}$ & $-\frac{\sqrt{5} v}{12 f^{2}}$ \\
$h \omega^{0} \omega^{0}$ & $\frac{v}{12 f^{2}}$ \\
$h \omega^{+} \omega^{-}$ & $\frac{v}{12 f^{2}}$ \\
\hline
\end{tabular}

Table 5. Scalar-Scalar-Vector and Scalar-Scalar-Scalar couplings at $\mathcal{O}\left(v^{2} / f^{2}\right)$. Other combinations also involving $h$ vanish.

gain intuition for the parametric dependence, first there is the universal one loop-factor $\left(16 \pi^{2}\right)^{-1}$. In addition, since we can always assume without loss of generality that the SM charged lepton Yukawa couplings are diagonal, any LFV Higgs decays must proceed via higher dimensional operators which are suppressed by the scale of new physics $f$ and scale like $v^{2} / f^{2}$. Moreover, the LFV Higgs decays must be proportional to the SM Yukawa couplings $\lambda$ and to the heavy lepton source of flavor violation $\Delta \kappa^{2} \sin 2 \theta$ because it is the 
misalignment of both sets of couplings which leads to LFV in the Higgs decay. ${ }^{12}$ Taking this into account and neglecting integral functions of mass ratios of heavy particles which are $\mathcal{O}(1)$ for masses not much larger than $f,{ }^{13}$ the amplitude for the Higgs decay $h \rightarrow \bar{\ell} \ell^{\prime}$ scales as

$$
\mathcal{M} \propto \frac{1}{16 \pi^{2}} \frac{v^{2}}{f^{2}} \lambda \Delta \kappa^{2} \sin 2 \theta .
$$

The two main results of this paper are then first to prove the finiteness of one-loop $h \rightarrow \bar{\ell} \ell^{\prime}$ decays in the LHT. As we show below, this relies on non-trivial cancellations among contributions from the heavy mirror and partner T-odd leptons. Second, the identification of new sources of LFV present in this class of models which contribute to LFV Higgs decays, as well as in general to all LFV amplitudes in the LHT.

\subsection{One-loop contribution of T-odd particles to $h \rightarrow \bar{\ell} \ell^{\prime}$ in the LHT}

We work in the renormalizable 't Hooft-Feynman gauge. This process shows significant differences with the corresponding gauge boson mediated processes $Z, \gamma \rightarrow \bar{\ell} \ell^{\prime}$. First, its finiteness requires the exchange of the full set of T-odd particles in the scalar and lepton sectors of the model introduced in the former section. This is apparent from inspection of the divergent contributions to the $h \rightarrow \bar{\ell} \ell^{\prime}$ amplitude. The different topologies are depicted in figure $1 .{ }^{14}$ Individually these amplitudes produce infinite and finite pieces at $\mathcal{O}(1)$. This means that they are suppressed only by the one-loop factor as well as Yukawa couplings and mixing angles, but not $v^{2} / f^{2}$. The total sum however cancels as it must since there is no available counterterm. Using the Feynman rules in the previous section one finds the divergent contributions listed in table $6 .{ }^{15}$ The numbers $C_{\mathrm{UV}}^{(1)}$ are the coefficients, up to a global factor, of $\frac{1}{\epsilon}$, with $\epsilon=4-d$ the extra dimensions in dimensional regularization. The dashes mean that the fields in the row do not close the loop of the topology in the column. The dots stand for the vanishing of the infinite and finite pieces of the corresponding diagrams. As indicated by the bullets, the sums of the different topologies in the last column give not only finite but vanishing contributions (the sum of the contributions with the same topology gathered in the last row are non-zero and infinite in general, but their total sum does cancel).

The $\mathcal{O}\left(v^{2} / f^{2}\right)$ contributions are more interesting. Again there is no counterterm for the corresponding operator of dimension 6 indicating that amplitude must be finite which we check explicitly. In table 7 we gather the coefficients $C_{\mathrm{UV}}^{\left(\frac{v^{2}}{f^{2}}\right)}$ of the divergent pieces for the different field contributions (rows) to a corresponding topology (columns). The notation is

\footnotetext{
${ }^{12}$ The Yukawa couplings $\kappa$ giving large masses to T-odd particles must enter squared because only T-odd particles run in the loop. In fact, it is their difference $\Delta \kappa^{2}$ that enters, whereas $\sin 2 \theta$ parameterizes the misalignment.

${ }^{13}$ As we discuss below, for very large masses of the partner lepton doublets these finite integrals can manifest a logarithmic behavior.

${ }^{14}$ There are new topologies with non-renormalizable couplings in this case, compared with the corresponding gauge transitions [26].

${ }^{15}$ As shown in next section, the divergent part of the amplitude can be written $\mathcal{M}_{\text {div }}\left(h \rightarrow \bar{\ell} \ell^{\prime}\right)=$ $\frac{1}{16 \pi^{2}}\left(C_{\mathrm{UV}}^{(1)}+\frac{v^{2}}{f^{2}} C_{\mathrm{UV}}^{\left(\frac{v^{2}}{f^{2}}\right)}\right) \frac{1}{\epsilon} \sum_{i=1}^{3} V_{\ell^{\prime} i}^{\dagger} V_{i \ell} \frac{m_{\ell_{H i}}^{2}}{f^{2}} \bar{u}\left(p^{\prime}, m_{\ell^{\prime}}\right)\left(\frac{m_{\ell^{\prime}}}{v} P_{L}+\frac{m_{\ell}}{v} P_{R}\right) v\left(p, m_{\ell}\right)$
} 


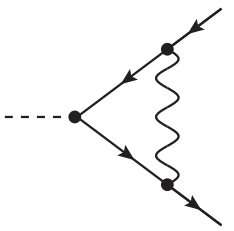

I
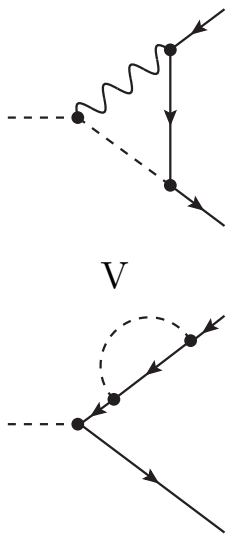

IX

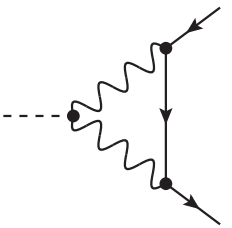

II

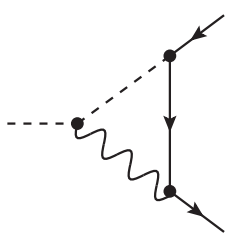

VI

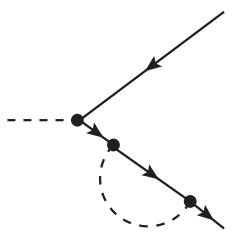

$\mathrm{X}$

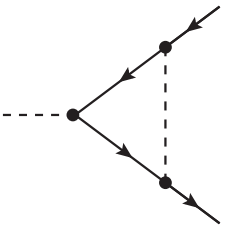

III

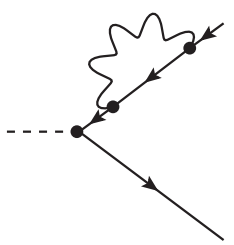

VII

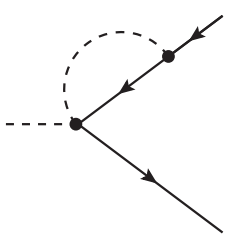

XI

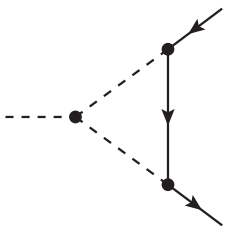

IV

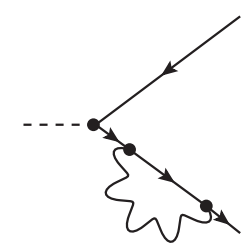

VIII

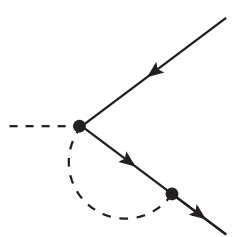

XII

Figure 1. Topologies contributing to $h \rightarrow \bar{\ell} \ell^{\prime}$.

\begin{tabular}{|r|cccccccc|c|}
\hline$C_{\mathrm{UV}}^{(1)}$ & $\mathrm{I}$ & $\mathrm{II}$ & $\mathrm{III}$ & $\mathrm{IV}$ & $\mathrm{V}+\mathrm{VI}$ & $\mathrm{VII}+\mathrm{VIII}$ & $\mathrm{IX}+\mathrm{X}$ & $\mathrm{XI}+\mathrm{XII}$ & $\mathrm{Sum}$ \\
\hline$\omega, \nu_{H}$ & - & - & $\bullet$ & $\bullet$ & - & - & 1 & -1 & $\bullet$ \\
$\omega^{0}, \ell_{H}$ & - & - & $\bullet$ & $\bullet$ & - & - & $\frac{1}{2}$ & $-\frac{1}{2}$ & $\bullet$ \\
$\eta, \ell_{H}$ & - & - & $\bullet$ & $\bullet$ & - & - & $\frac{1}{10}$ & $-\frac{1}{10}$ & $\bullet$ \\
\hline Total & - & - & $\bullet$ & $\bullet$ & - & - & $\frac{8}{5}$ & $-\frac{8}{5}$ & $\bullet$ \\
\hline
\end{tabular}

Table 6. Divergent contributions proportional to $\frac{1}{\epsilon}$, with $\epsilon=4-d$ the extra dimensions in dimensional regularization, of each particle set running in the loop and topology in figure 1 contributing at $\mathcal{O}(1)$. A dash means that the field set does not run in the diagram, whereas a dot indicates that the infinite and finite parts vanish.

as in table 6 , but now a 0 means that only the infinite piece cancels. As can be observed by summing the entries of the last column, which adds to zero, the contribution of the charged diagrams (those exchanging heavy neutrinos) is finite. It is clear however from examining the table, and looking for example at the $\omega, \nu_{H}$ and $\Phi, \tilde{\nu}^{c}, \nu_{H}$ contributions to the III topology, that the scalar triplet of hypercharge 1 as well as the additional vector-like partner lepton doublets have to be taken into account to obtain a finite result. This was perhaps expected since the electroweak triplets and partner leptons are needed in order to guarantee that the $\mathrm{SO}(5)$ global symmetry is preserved. Quite often however it has been assumed that they are heavy enough to be ignored, that they can be decoupled. This is true for gauge boson mediated LFV processes where chiral symmetry allows for the $\Phi$ and 


\begin{tabular}{|c|c|c|c|c|c|c|c|c|c|}
\hline$C_{\mathrm{UV}}^{\left(\frac{v^{2}}{f^{2}}\right)}$ & I & II & III & IV & $\mathrm{V}+\mathrm{VI}$ & VII+VIII & $\mathrm{IX}+\mathrm{X}$ & $\mathrm{XI}+\mathrm{XII}$ & Sum \\
\hline$W_{H}, \nu_{H}$ & 0 & 0 & - & - & - & $\bullet$ & - & - & 0 \\
\hline$W_{H}, \omega, \nu_{H}$ & - & - & - & - & 0 & - & - & - & 0 \\
\hline$\omega, \nu_{H}$ & - & - & $\frac{1}{4}$ & $-\frac{1}{8}$ & - & - & $-\frac{1}{6}$ & $\frac{5}{24}$ & $\frac{1}{6}$ \\
\hline$Z_{H}, \ell_{H}$ & $\bullet$ & 0 & - & - & - & $\bullet$ & - & - & 0 \\
\hline$Z_{H}, \omega^{0}, \ell_{H}$ & - & - & - & - & 0 & - & - & - & 0 \\
\hline$\omega^{0}, \ell_{H}$ & - & - & $\bullet$ & $-\frac{1}{16}$ & - & - & $-\frac{13}{48}+x_{H} \frac{c_{W}}{s_{W}}$ & $\frac{7}{16}-x_{H} \frac{c_{W}}{s_{W}}$ & $\frac{5}{48}$ \\
\hline$A_{H} \ell_{H}$ & $\bullet$ & 0 & - & - & - & $\bullet$ & - & - & 0 \\
\hline$A_{H}, \eta, \ell_{H}$ & - & - & - & - & 0 & - & - & - & 0 \\
\hline$\eta, \ell_{H}$ & - & - & $\bullet$ & $-\frac{1}{16}$ & - & - & $-\frac{23}{240}-x_{H} \frac{s_{W}}{5 c_{W}}$ & $-\frac{17}{240}+x_{H} \frac{s_{W}}{5 c_{W}}$ & $-\frac{11}{48}$ \\
\hline$Z_{H}, A_{H}, \ell_{H}$ & - & 0 & - & - & - & - & - & - & 0 \\
\hline$\omega^{0}, \eta, \ell_{H}$ & - & - & - & $\frac{1}{8}$ & - & - & - & - & $\frac{1}{8}$ \\
\hline$W_{H}, \Phi, \nu_{H}$ & - & - & - & - & 0 & - & - & - & 0 \\
\hline$\Phi, \nu_{H}$ & - & - & $\bullet$ & $\bullet$ & - & - & $-\frac{1}{8}$ & $\frac{1}{24}$ & $-\frac{1}{12}$ \\
\hline$\omega, \Phi, \nu_{H}$ & - & - & - & $\frac{1}{6}$ & - & - & - & - & $\frac{1}{6}$ \\
\hline$\omega^{0}, \Phi^{P}, \ell_{H}$ & - & - & - & $\frac{1}{24}$ & - & - & - & - & $\frac{1}{24}$ \\
\hline$\eta, \Phi^{P}, \ell_{H}$ & - & - & - & $-\frac{1}{24}$ & - & - & - & - & $-\frac{1}{24}$ \\
\hline$\Phi, \tilde{\nu}^{c}, \nu_{H}$ & - & - & $-\frac{1}{4}$ & $\frac{1}{24}$ & - & - & - & $-\frac{1}{24}$ & $-\frac{1}{4}$ \\
\hline Total & 0 & 0 & 0 & $\frac{1}{12}$ & 0 & $\bullet$ & $-\frac{49}{120}$ & $\frac{39}{120}$ & 0 \\
\hline
\end{tabular}

Table 7. As in table 6 but to $\mathcal{O}\left(v^{2} / f^{2}\right) . x_{H}=\frac{5 t_{W}}{4\left(5-t_{W}^{2}\right)}$ is defined in eq. (2.32) with $t_{W}=\frac{s_{W}}{c_{W}}$.

$\tilde{\nu}^{c}$ contributions to be decoupled without introducing a divergence, but clearly does not hold for $h \rightarrow \tau \mu$. We will further discuss the $Z, \gamma \rightarrow \bar{\ell} \ell^{\prime}$ amplitudes elsewhere.

\subsection{New sources of flavor violation contributing to $h \rightarrow \bar{\ell} \ell^{\prime}$ in the LHT}

We work out the finite part in this subsection to discuss the sources of LFV in the LHT, and their behavior in the non-decoupling limit. ${ }^{16}$ These sources also contribute to other processes like $Z, \gamma \rightarrow \bar{\ell} \ell^{\prime}$, already discussed in the literature [26, 39], though only the approximation of decoupled partner leptons and small momenta. These assumptions can not be made in general and in particular for Higgs decays. First, as shown in the previous subsection, the partner leptons are needed to make $h \rightarrow \bar{\ell} \ell^{\prime}$ finite. The fact that the partner lepton mass, which introduces a soft breaking of the global $\mathrm{SO}(5)$, can not be taken to infinity and decoupled manifests as a logarithmic dependence of the $h \rightarrow \bar{\ell} \ell^{\prime}$ amplitude on its mass, as shown below. Second, for on and off shell Higgs and $Z$ decays at the LHC there will be significant transfer of momenta. Thus a global fit using the complete expressions for the LFV gauge boson mediated processes is also necessary [38], but we focus here first on the $h \rightarrow \tau \mu$ decay.

\footnotetext{
${ }^{16}$ We have validated our calculation using the Mathematica package FeynCalc [43, 44].
} 
Using the Feynman rules in the previous section, we calculate the one-loop contributions of the T-odd gauge bosons, scalars and fermions to the $h \rightarrow \bar{\ell} \ell^{\prime}$ amplitude in the LHT. This can be written as

$$
\mathcal{M}\left(h \rightarrow \bar{\ell} \ell^{\prime}\right)=\bar{u}\left(p^{\prime}, m_{\ell^{\prime}}\right)\left(\frac{m_{\ell^{\prime}}}{v} c_{L}^{\ell \ell^{\prime}} P_{L}+\frac{m_{\ell}}{v} c_{R}^{\ell \ell^{\prime}} P_{R}\right) v\left(p, m_{\ell}\right),
$$

with

$$
\begin{aligned}
c_{L(R)}^{\ell \ell^{\prime}}=\frac{\alpha_{W}}{4 \pi} \frac{v^{2}}{f^{2}} & {\left[\sum_{i=1}^{3} V_{\ell^{\prime} i}^{\dagger} V_{i \ell} F\left(m_{\ell_{H i}}, M_{W_{H}}, M_{A_{H}}, M_{\Phi}\right)\right.} \\
& \left.\sum_{i, j, k=1}^{3} V_{\ell^{\prime} i}^{\dagger} \frac{m_{\ell_{H i}}}{M_{W_{H}}} W_{i j}^{\dagger} W_{j k} \frac{m_{\ell_{H k}}}{M_{W_{H}}} V_{k \ell} G\left(m_{\tilde{\nu}_{j}^{c}}, m_{\ell_{H k(i)}}, M_{\Phi}\right)\right] .
\end{aligned}
$$

Note the different index contraction for $c_{L}^{\ell \ell^{\prime}}$ and $c_{R}^{\ell \ell^{\prime}}$, and the mass relations in eqs. (2.33) and (2.39) between $M_{W_{H}}$ and $M_{Z_{H}}$ and between $m_{\ell_{H i}}$ and $m_{\nu_{H i}}$, respectively. The function $F$ is more involved and worked out in the appendix. Making use of the scalar integrals of three-point functions in ref. [45] (we omit the first three arguments of the three-point functions, which we take equal to 0 for the external fermion momenta and to $Q^{2}=M_{h}^{2}$ for the Higgs momentum), the function $G$ in the second term reduces to

$$
\begin{aligned}
G\left(m_{\tilde{\nu}_{j}^{c}}, m_{\ell_{H k}}, M_{\Phi}\right)= & \frac{1}{16}-\frac{1}{2} C_{00}\left(M_{\Phi}^{2}, m_{\tilde{\nu}_{j}^{c}}^{2}, m_{\ell_{H k}}^{2}\right) \\
& -\frac{1}{8} m_{\tilde{\nu}_{j}^{c}} m_{\ell_{H k}} C_{0}\left(M_{\Phi}^{2}, m_{\tilde{\nu}_{j}^{c}}^{2}, m_{\ell_{H k}}^{2}\right) \\
& -\frac{1}{12} M_{\Phi}^{2} C_{1}\left(m_{\tilde{\nu}_{j}^{c}}^{2}, M_{\Phi}^{2}, M_{\Phi}^{2}\right) .
\end{aligned}
$$

As is apparent from eq. (3.3), there are two sources of LFV present in the LHT model. They are proportional to the mixing matrices $V$ and $W$ in eq. (2.40) parameterizing the misalignment of the light lepton doublets with the heavy (vector-like) ones. The unitary matrix $V$ describes the flavor rotation between the former and their T-odd (left-handed) doublet (mirror) partners. The unitary matrix $W$ parameterizes flavor rotations between the right-handed doublet counterparts of the latter and the extra doublets required to give the partner leptons a mass.

The two sources of LFV in eq. (3.3) have a different dependence on the masses of the heavy vector-like leptons. The first source coming from the misalignment of the mirror leptons and involving only the rotation matrix $V$ depend on the mirror lepton masses, but not on the partner lepton masses, and only decouples when the scale $f$ is taken large. In contrast, the second source of LFV resulting from the misalignment of the additional lepton doublets and involving both rotation matrices $V$ and $W$ is also function of the extra partner lepton masses $m_{\tilde{l}_{i}}$, which have a different origin and may be a priori much larger than the scale $f$. In this limit this contribution grows logarithmically, reflecting the need for further NP to provide these leptons a mass. The behavior in this limit is dictated by the second term in eq. (3.4) proportional to $C_{00}$ and coming from topology III with particle content $\Phi, \tilde{\nu}^{c}, \nu_{H}$. This term grows as $\ln m_{\tilde{\nu}_{j}^{c}}$ and hence, there is no decoupling. 


\section{Model dependent limits on the Higgs coupling to a pair of different leptons in the LHT}

We now estimate the corresponding branching ratio for the most interesting experimental channel $h \rightarrow \tau^{+} \mu^{-}+\tau^{-} \mu^{+}$. To this end we still have to correct for the final mass eigenstates. Indeed, the contributions to $h \rightarrow \bar{\ell} \ell^{\prime}$ in eq. (3.2) imply that the corresponding off-diagonal entries $\ell \ell^{\prime}$ of the charged lepton mass matrix also receive one-loop corrections with the Higgs field insertion replaced by the vev. Hence, a further diagonalization of this mass matrix is required to obtain the lepton mass eigenstates at the order in which we work. This diagonalization and correction has been discussed in ref. [46] for the quark sector (see also ref. [47]), and amounts to an extra multiplicative factor $2 / 3$ for the amplitude in eq. (3.2) for the actual final lepton mass eigenstates. ${ }^{17}$

This factor $2 / 3$ can be easily understood as follows. At order $v^{2} / f^{2}$ we can completely describe the SM charged lepton masses and their couplings to the Higgs boson by means of the following effective Lagrangian, written in the basis defined by eq. (2.37),

$$
\begin{aligned}
\mathcal{L}_{\text {eff }} & =-\frac{\sqrt{2}}{v} m_{\ell_{i}} \overline{l_{L i}} \phi \ell_{R i}+\frac{c_{i j}}{f^{2}}|\phi|^{2} \overline{l_{L i}} \phi \ell_{R j}+\text { h.c. }+\ldots \\
& =\left[\left(-m_{\ell_{i}} \delta_{i j}+\frac{1}{2 \sqrt{2}} \frac{v^{3}}{f^{2}} c_{i j}\right)+\frac{h}{v}\left(-m_{\ell_{i}} \delta_{i j}+\frac{3}{2 \sqrt{2}} \frac{v^{3}}{f^{2}} c_{i j}\right)\right] \overline{\ell_{L i}} \ell_{R j}+\text { h.c. }+\ldots,
\end{aligned}
$$

where $c_{i j}$ are the corresponding (one-loop) Wilson coefficients. The key point is the relative factor of 3 between the Yukawa coupling and the mass term at order $v^{2} / f^{2}$, originating from the expansion $(v+h)^{3}=v^{3}+3 v^{2} h+\ldots$ in the dimension 6 operator above. Due to this factor the mass and Yukawa matrices are no longer proportional to each other and diagonalizing the former does not automatically diagonalize the latter. We can go to the physical basis by means of the usual bi-unitary transformation

$$
\ell_{L, R i}=\left(U_{L, R}\right)_{i j} \ell_{L, R j}^{p h y s}
$$

where we have emphasized that $\ell_{L, R}^{\text {phys }}$ are the charged leptons in the physical basis and $U_{L, R}$ are $3 \times 3$ unitary matrices that can be written, up to order $v^{2} / f^{2}$, as

$$
U_{L, R}=1+\frac{v^{2}}{f^{2}} A_{L, R}
$$

with $A_{L, R}$ antihermitian matrices. The explicit form of these matrices can be found in [46] but it is not needed for the discussion of the off-diagonal terms. Then, the condition that the mass matrix is diagonal in the physical basis in particular requires that the coefficients of the off-diagonal terms of order $v^{2} / f^{2}$ cancel in this basis

$$
\frac{c_{i j} v}{2 \sqrt{2}}+\left(A_{L}\right)_{i j} m_{\ell_{j}}-m_{\ell_{i}}\left(A_{R}\right)_{i j}=0, \quad(i \neq j, \text { physical basis })
$$

\footnotetext{
${ }^{17}$ In contrast, for the gauge couplings the effect of this final rotation is higher order (for the neutral current couplings) or physically unobservable (for the charged current ones).
} 
which in turn implies, for the off-diagonal contribution to the Yukawa coupling,

$$
\begin{aligned}
\frac{v^{2}}{f^{2}}\left[\frac{3 c_{i j}}{2 \sqrt{2}}+\left(A_{L}\right)_{i j} \frac{m_{\ell_{j}}}{v}\right. & \left.-\frac{m_{\ell_{i}}}{v}\left(A_{R}\right)_{i j}\right] h \overline{h \bar{\ell}_{L i}} \ell_{R j}+\ldots \\
& =\frac{1}{\sqrt{2}} \frac{v^{2}}{f^{2}} c_{i j} h \overline{\ell_{L i}} \ell_{R j}+\ldots, \quad(i \neq j, \text { physical basis }) .
\end{aligned}
$$

Thus we see that the effect of going to the physical basis just amounts to a simple re-scaling of the off-diagonal Yukawa couplings by a factor $2 / 3$.

The LFV partial width can therefore be written as

$$
\Gamma\left(h \rightarrow \tau^{+} \mu^{-}+\tau^{-} \mu^{+}\right)=\frac{M_{h}}{16 \pi} \frac{m_{\tau}^{2}+m_{\mu}^{2}}{v^{2}} \frac{4}{9}\left(\left|c_{L}^{\tau \mu}\right|^{2}+\left|c_{R}^{\tau \mu}\right|^{2}\right),
$$

and its branching ratio

$$
\operatorname{Br}(h \rightarrow \tau \mu)=\operatorname{Br}(h \rightarrow b \bar{b}) \frac{\Gamma\left(h \rightarrow \tau^{+} \mu^{-}+\tau^{-} \mu^{+}\right)}{\Gamma(h \rightarrow b \bar{b})} \simeq 0.6 \frac{m_{\tau}^{2}}{6 m_{b}^{2}} \frac{4}{9}\left(\left|c_{L}^{\tau \mu}\right|^{2}+\left|c_{R}^{\tau \mu}\right|^{2}\right) .
$$

Now, using eq. (3.3), with the mixing matrices (the $V$ columns correspond to $e, \mu$ and $\tau$, respectively)

$$
V=\left(\begin{array}{ccc}
1 & 0 & 0 \\
0 & \cos \theta_{1} & \sin \theta_{1} \\
0 & -\sin \theta_{1} & \cos \theta_{1}
\end{array}\right), \quad W=\left(\begin{array}{ccc}
1 & 0 & 0 \\
0 & \cos \theta_{2} & \sin \theta_{2} \\
0 & -\sin \theta_{2} & \cos \theta_{2}
\end{array}\right),
$$

we obtain

$$
\operatorname{Br}(h \rightarrow \tau \mu) \simeq 0.2 \times 10^{-6},
$$

for $f=1 \mathrm{TeV}$, fixing the heavy gauge boson masses $M_{W_{H}, Z_{H}, A_{H}}$ in eq. (2.33) and $M_{\Phi} \simeq$ $\sqrt{2} M_{h} f / v$ [48], and $m_{\ell_{H 2,3}}=1.0,8.1 \mathrm{TeV}, m_{\tilde{l}_{2,3}}=10,50 \mathrm{TeV}$, and $\theta_{1,2}=\frac{\pi}{3}, \frac{\pi}{25}$, respectively. In general, the LFV Higgs branching ratios tend to be smaller when $m_{\tilde{l}_{i}} \sim m_{\ell_{H i}} \sim M_{W_{H}}$ and there are often large cancellations. At any rate, in order to assess the experimentally allowed regions in parameter space in the LHT these predictions have to be confronted with the corresponding ones with gauge bosons, and all of them with the stringent experimental limits on LFV processes. We will present such a detailed study elsewhere [38].

\section{$5 \quad$ Summary and conclusions}

We have calculated loop induced lepton flavor violating Higgs decays in the Littlest Higgs model with T-parity including all contributions from the T-odd lepton sector. We have shown that a finite amplitude is obtained only when all of these contributions are included in contrast to lepton flavor violating processes mediated by gauge bosons where the partners of the right-handed mirror leptons can be decoupled from the spectrum. These partners are necessary to cancel the divergence in the Higgs mass introduced by the mirror leptons but are otherwise unnecessary and assumed to be decoupled in previous phenomenological studies. We have emphasized that these partner leptons can not be decoupled in Higgs 
decays and furthermore, they introduce a new source of lepton flavor violation via their couplings to the physical pseudo-Goldstone electroweak triplet scalar.

Although this extra source also affects lepton flavor violating processes mediated by gauge bosons, it decouples from these amplitudes in the limit of heavy mass for the partner leptons. However, if all the partner leptons are kept at the same order as the other T-odd particles, all their contributions are expected to be of similar size. This implies that the contributions of partner leptons as well as the pseudo-Goldstone scalar electroweak triplet must also be taken into account when estimating LFV processes mediated by photons and $Z$ bosons. Moreover, an assessment about the parameter space of this model allowed by experiment requires the calculation of these new contributions and to perform a global fit to all LFV processes experimentally accessible. A detailed discussion of the corresponding limits will be presented elsewhere.

Barring these further constraints, we find that the $h \rightarrow \tau \mu$ branching ratio can be as large as $\sim 0.2 \times 10^{-6}$ for large mixings and all T-odd particle masses of the order a few TeV.

\section{Acknowledgments}

We thank useful discussions and comments by A. David, M. Masip, R. Pittau, J. Wudka and the anonymous referee. This work has been supported in part by the European Commission through the contract PITN-GA-2012-316704 (HIGGSTOOLS), by the Ministry of Economy, Industry and Competitiveness (MINEICO), under grant numbers FPA201347836-C3-1,2,3-P and FPA2016-78220-C3-1,2,3-P (fondos FEDER), and by the Junta de Andalucía grant FQM 101. P.T. is partially supported by FPA2013-46570.

\section{A Expressions for the amplitudes}

The function $F$ in eq. (3.3) can be split depending on the fields running in the loop and their common mass dependence in 8 pieces corresponding to the first 8 row groupings in table 6 , separated by horizontal lines: ${ }^{18}$

$$
F=\left.F\right|_{W_{H}}+\left.F\right|_{Z_{H}}+\left.F\right|_{A_{H}}+\left.F\right|_{Z_{H} A_{H}}+\left.F\right|_{\Phi}+\left.F\right|_{\omega \Phi}+\left.F\right|_{\omega^{0} \Phi^{P}}+\left.F\right|_{\eta \Phi^{P}}
$$

Using the scalar integrals of two and three-point functions in ref. [45] (see also ref. [26] for notation; in particular, we omit the first three arguments of the three-point functions, which we take equal to 0 for the external fermion momenta and to $Q^{2}=M_{h}^{2}$ for the Higgs momentum), these contributions read

$$
\begin{aligned}
\left.F\right|_{W_{H}}= & -\frac{1}{16}-\frac{1}{16} \frac{m_{\ell_{H i}}^{2}}{M_{W_{H}}^{2}}+\frac{1}{2} C_{00}\left(m_{\ell_{H i}}^{2}, M_{W_{H}}^{2}, M_{W_{H}}^{2}\right)+\frac{m_{\ell_{H i}}^{2}}{24} C_{0}\left(m_{\ell_{H i}}^{2}, M_{W_{H}}^{2}, M_{W_{H}}^{2}\right) \\
& -\frac{1}{2} \frac{m_{\ell_{H i}}^{2}}{M_{W_{H}}^{2}}\left[\frac{1}{12} B_{0}\left(0 ; M_{W_{H}}^{2}, m_{\ell_{H i}}^{2}\right)-C_{00}\left(M_{W_{H}}^{2}, m_{\ell_{H i}}^{2}, m_{\ell_{H i}}^{2}\right)\right]
\end{aligned}
$$

\footnotetext{
${ }^{18}$ Below, we use $m_{\ell_{H i}}$ everywhere, although in the (charged) diagrams exchanging mirror neutrinos, $m_{\nu_{H i}}$ must be understood. (Similarly to the case of the function $G$ in eq. (3.4), where the relevant mass is $m_{\tilde{\nu}_{j}^{c}}$, when applicable.) Analogously, we denote by $M_{\Phi}$ the mass of any scalar triplet component, although they can differ by a small amount $\mathcal{O}\left(v^{2} / f^{2}\right)$ after EWSB, which we can neglect at the order we work.
} 


$$
\begin{aligned}
& +\frac{m_{\ell_{H i}}^{2}}{2}\left[\left(\frac{1}{3}+\frac{M_{W_{H}}^{2}}{m_{\ell_{H i}}^{2}}\right) C_{1}\left(m_{\ell_{H i}}^{2}, M_{W_{H}}^{2}, M_{W_{H}}^{2}\right)\right. \\
& \left.-\left(1+\frac{1}{2} \frac{m_{\ell_{H i}}^{2}}{M_{W_{H}}^{2}}\right) C_{1}\left(M_{W_{H}}^{2}, m_{\ell_{H i}}^{2}, m_{\ell_{H i}}^{2}\right)-\frac{1}{2} C_{0}\left(M_{W_{H}}^{2}, m_{\ell_{H i}}^{2}, m_{\ell_{H i}}^{2}\right)\right], \\
& \left.F\right|_{Z_{H}}=-\frac{1}{32}+\frac{1}{4} C_{00}\left(m_{\ell_{H i}}^{2}, M_{W_{H}}^{2}, M_{W_{H}}^{2}\right)+\frac{1}{48} m_{\ell_{H i}}^{2} C_{0}\left(m_{\ell_{H i}}^{2}, M_{W_{H}}^{2}, M_{W_{H}}^{2}\right) \\
& -\frac{1}{24} \frac{m_{\ell_{H i}}^{2}}{M_{W_{H}}^{2}}\left[\frac{1}{2} B_{1}\left(0 ; m_{\ell_{H i}}^{2}, M_{W_{H}}^{2}\right)-B_{0}\left(0 ; M_{W_{H}}^{2}, m_{\ell_{H i}}^{2}\right)\right] \\
& +\left(\frac{1}{8} M_{W_{H}}^{2}+\frac{1}{12} m_{\ell_{H i}}^{2}\right) C_{1}\left(m_{\ell_{H i}}^{2}, M_{W_{H}}^{2}, M_{W_{H}}^{2}\right) \\
& \left.F\right|_{A_{H}}=-\frac{1}{4} \frac{M_{A_{H}}^{2}}{M_{W_{H}}^{2}}\left[\frac{1}{8}-C_{00}\left(m_{\ell_{H i}}^{2}, M_{A_{H}}^{2}, M_{A_{H}}^{2}\right)-\frac{1}{12} m_{\ell_{H i}}^{2} C_{0}\left(m_{\ell_{H i}}^{2}, M_{A_{H}}^{2}, M_{A_{H}}^{2}\right)\right] \\
& -\frac{1}{8} \frac{m_{\ell_{H i}}^{2}}{M_{W_{H}}^{2}}\left[\frac{1}{6} B_{1}\left(0 ; M_{A_{H}}^{2}, m_{\ell_{H i}}^{2}\right)+B_{0}\left(0 ; M_{A_{H}}^{2}, m_{\ell_{H i}}^{2}\right)\right] \\
& +\frac{M_{A_{H}}^{2}}{M_{W_{H}}^{2}}\left(\frac{1}{8} M_{A_{H}}^{2}+\frac{1}{12} m_{\ell_{H i}}^{2}\right) C_{1}\left(m_{\ell_{H i}}^{2}, M_{A_{H}}^{2}, M_{A_{H}}^{2}\right), \\
& \left.F\right|_{Z_{H} A_{H}}=\frac{1}{24} \frac{m_{\ell_{H i}}^{2}}{M_{W_{H}}^{2}}\left[\frac{1}{2} B_{1}\left(0 ; M_{W_{H}}^{2}, m_{\ell_{H i}}^{2}\right)+\frac{1}{2} B_{1}\left(0 ; M_{A_{H}}^{2}, m_{\ell_{H i}}^{2}\right)+B_{0}\left(0 ; M_{W_{H}}^{2}, m_{\ell_{H i}}^{2}\right)\right. \\
& \left.+B_{0}\left(0 ; M_{A_{H}}^{2}, m_{\ell_{H i}}^{2}\right)\right]-\frac{1}{4} M_{A_{H}}^{2} C_{1}\left(m_{\ell_{H i}}^{2}, M_{W_{H}}^{2}, M_{A_{H}}^{2}\right) \\
& +\frac{m_{\ell_{H i}}^{2}}{M_{W_{H}}^{2}}\left(\frac{M_{W_{H}}^{2}}{24}+\frac{M_{A_{H}}^{2}}{24}\right)\left[C_{1}\left(m_{\ell_{H i}}^{2}, M_{W_{H}}^{2}, M_{A_{H}}^{2}\right)+C_{0}\left(m_{\ell_{H i}}^{2}, M_{W_{H}}^{2}, M_{A_{H}}^{2}\right)\right] \\
& \left.F\right|_{\Phi}=\frac{1}{16}-\frac{1}{2} C_{00}\left(m_{\ell_{H i}}^{2}, M_{\Phi}^{2}, M_{\Phi}^{2}\right)-\frac{1}{24} \frac{m_{\ell_{H i}}^{2}}{M_{W_{H}}^{2}} B_{0}\left(0 ; M_{\Phi}^{2}, m_{\ell_{H i}}^{2}\right), \\
& \left.F\right|_{\omega \Phi}=\frac{1}{24} \frac{m_{\ell_{H i}}^{2}}{M_{W_{H}}^{2}}\left[B_{0}\left(0 ; M_{W_{H}}^{2}, m_{\ell_{H i}}^{2}\right)+B_{0}\left(0 ; M_{\Phi}^{2}, m_{\ell_{H i}}^{2}\right)\right. \\
& \left.+\left(M_{W_{H}}^{2}+M_{\Phi}^{2}\right) C_{0}\left(m_{\ell_{H i}}^{2}, M_{W_{H}}^{2}, M_{\Phi}^{2}\right)\right], \\
& \left.F\right|_{\omega^{0} \Phi^{P}}=\frac{1}{96} \frac{m_{\ell_{H i}}^{2}}{M_{W_{H}}^{2}}\left[B_{0}\left(0 ; M_{W_{H}}^{2}, m_{\ell_{H i}}^{2}\right)+B_{0}\left(0 ; M_{\Phi}^{2}, m_{\ell_{H i}}^{2}\right)\right. \\
& \left.+\left(M_{W_{H}}^{2}+M_{\Phi}^{2}\right) C_{0}\left(m_{\ell_{H i}}^{2}, M_{W_{H}}^{2}, M_{\Phi}^{2}\right)\right], \\
& \left.F\right|_{\eta \Phi^{P}}=-\frac{1}{96} \frac{m_{\ell_{H i}}^{2}}{M_{W_{H}}^{2}}\left[B_{0}\left(0 ; M_{A_{H}}^{2}, m_{\ell_{H i}}^{2}\right)+B_{0}\left(0 ; M_{\Phi}^{2}, m_{\ell_{H i}}^{2}\right)\right. \\
& \left.+\left(M_{A_{H}}^{2}+M_{\Phi}^{2}\right) C_{0}\left(m_{\ell_{H i}}^{2}, M_{A_{H}}^{2}, M_{\Phi}^{2}\right)\right] .
\end{aligned}
$$

We neglect terms proportional to $Q^{2}=M_{h}^{2}$ because they are next order in $v^{2} / f^{2}$. Corrections proportional to light (SM) lepton masses are also neglected everywhere. 
Open Access. This article is distributed under the terms of the Creative Commons Attribution License (CC-BY 4.0), which permits any use, distribution and reproduction in any medium, provided the original author(s) and source are credited.

\section{References}

[1] CMS collaboration, Observation of a new boson at a mass of $125 \mathrm{GeV}$ with the CMS experiment at the LHC, Phys. Lett. B 716 (2012) 30 [arXiv:1207.7235] [INSPIRE].

[2] ATLAS collaboration, Observation of a new particle in the search for the Standard Model Higgs boson with the ATLAS detector at the LHC, Phys. Lett. B 716 (2012) 1 [arXiv: 1207.7214] [INSPIRE].

[3] A. Falkowski, F. Riva and A. Urbano, Higgs at last, JHEP 11 (2013) 111 [arXiv:1303.1812] [INSPIRE].

[4] CMS collaboration, Constraints on the spin-parity and anomalous HVV couplings of the Higgs boson in proton collisions at 7 and 8 TeV, Phys. Rev. D 92 (2015) 012004 [arXiv: 1411.3441] [INSPIRE].

[5] ATLAS, CMS collaboration, G. Aad et al., Measurements of the Higgs boson production and decay rates and constraints on its couplings from a combined ATLAS and CMS analysis of the LHC pp collision data at $\sqrt{s}=7$ and 8 TeV, JHEP 08 (2016) 045 [arXiv: 1606.02266] [INSPIRE].

[6] N. Arkani-Hamed, A.G. Cohen and H. Georgi, Electroweak symmetry breaking from dimensional deconstruction, Phys. Lett. B 513 (2001) 232 [hep-ph/0105239] [INSPIRE].

[7] M. Schmaltz and D. Tucker-Smith, Little Higgs review, Ann. Rev. Nucl. Part. Sci. 55 (2005) 229 [hep-ph/0502182] [INSPIRE].

[8] T. Han, H.E. Logan and L.-T. Wang, Smoking-gun signatures of little Higgs models, JHEP 01 (2006) 099 [hep-ph/0506313] [INSPIRE].

[9] M. Perelstein, Little Higgs models and their phenomenology, Prog. Part. Nucl. Phys. 58 (2007) 247 [hep-ph/0512128] [INSPIRE].

[10] H.-C. Cheng and I. Low, TeV symmetry and the little hierarchy problem, JHEP 09 (2003) 051 [hep-ph/0308199] [INSPIRE].

[11] H.-C. Cheng and I. Low, Little hierarchy, little Higgses and a little symmetry, JHEP 08 (2004) 061 [hep-ph/0405243] [INSPIRE].

[12] I. Low, T parity and the littlest Higgs, JHEP 10 (2004) 067 [hep-ph/0409025] [inSPIRE].

[13] J. Hubisz and P. Meade, Phenomenology of the littlest Higgs with T-parity, Phys. Rev. D 71 (2005) 035016 [hep-ph/0411264] [INSPIRE].

[14] J. Hubisz, P. Meade, A. Noble and M. Perelstein, Electroweak precision constraints on the littlest Higgs model with T parity, JHEP 01 (2006) 135 [hep-ph/0506042] [INSPIRE].

[15] C.G. Callan Jr., S.R. Coleman, J. Wess and B. Zumino, Structure of phenomenological Lagrangians. 2, Phys. Rev. 177 (1969) 2247 [INSPIRE].

[16] C.T. Hill and R.J. Hill, $T^{-}$parity violation by anomalies, Phys. Rev. D 76 (2007) 115014 [arXiv: 0705.0697] [INSPIRE]. 
[17] T. Han, H.E. Logan, B. Mukhopadhyaya and R. Srikanth, Neutrino masses and lepton-number violation in the littlest Higgs scenario, Phys. Rev. D 72 (2005) 053007 [hep-ph/0505260] [INSPIRE].

[18] J. Hubisz, S.J. Lee and G. Paz, The flavor of a little Higgs with T-parity, JHEP 06 (2006) 041 [hep-ph/0512169] [INSPIRE].

[19] C.-R. Chen, K. Tobe and C.P. Yuan, Higgs boson production and decay in little Higgs models with T-parity, Phys. Lett. B 640 (2006) 263 [hep-ph/0602211] [INSPIRE].

[20] M. Blanke, A.J. Buras, A. Poschenrieder, C. Tarantino, S. Uhlig and A. Weiler, Particle-antiparticle mixing, $\epsilon_{K}, \Delta \Gamma_{q}, A_{S L}^{q}, A_{C P}\left(B_{d} \rightarrow \psi K_{S}\right), A_{C P}\left(B_{s} \rightarrow \psi \phi\right)$ and $B \rightarrow X(s, d \gamma)$ in the littlest Higgs model with T-parity, JHEP 12 (2006) 003 [hep-ph/0605214] [INSPIRE].

[21] A.J. Buras, A. Poschenrieder, S. Uhlig and W.A. Bardeen, Rare $K$ and $B$ decays in the littlest Higgs model without $T^{-}$parity, JHEP 11 (2006) 062 [hep-ph/0607189] [INSPIRE].

[22] A. Belyaev, C.-R. Chen, K. Tobe and C.P. Yuan, Phenomenology of littlest Higgs model with $T^{-}$parity: including effects of $T^{-}$odd fermions, Phys. Rev. D 74 (2006) 115020 [hep-ph/0609179] [INSPIRE].

[23] C.T. Hill and R.J. Hill, Topological physics of little Higgs bosons, Phys. Rev. D 75 (2007) 115009 [hep-ph/0701044] [INSPIRE].

[24] X.-F. Han, L. Wang and J.M. Yang, Higgs and Z-boson FCNC decays correlated with B-meson decays in littlest Higgs model with T-parity, Phys. Rev. D 78 (2008) 075017 [arXiv:0807.4480] [INSPIRE].

[25] T. Goto, Y. Okada and Y. Yamamoto, Ultraviolet divergences of flavor changing amplitudes in the littlest Higgs model with T-parity, Phys. Lett. B 670 (2009) 378 [arXiv:0809.4753] [INSPIRE].

[26] F. del Aguila, J.I. Illana and M.D. Jenkins, Precise limits from lepton flavour violating processes on the Littlest Higgs model with T-parity, JHEP 01 (2009) 080 [arXiv:0811.2891] [INSPIRE].

[27] M. Blanke, A.J. Buras, B. Duling, S. Recksiegel and C. Tarantino, FCNC processes in the littlest Higgs model with T-parity: a 2009 look, Acta Phys. Polon. B 41 (2010) 657 [arXiv:0906.5454] [INSPIRE].

[28] F. del Aguila, J.I. Illana and M.D. Jenkins, Muon to electron conversion in the Littlest Higgs model with T-parity, JHEP 09 (2010) 040 [arXiv:1006.5914] [INSPIRE].

[29] T. Goto, Y. Okada and Y. Yamamoto, Tau and muon lepton flavor violations in the littlest Higgs model with T-parity, Phys. Rev. D 83 (2011) 053011 [arXiv:1012.4385] [INSPIRE].

[30] H.-S. Hou, H. Sun and Y.-J. Zhou, Flavor changing top quark decay and bottom-strange production in the littlest Higgs model with T-parity, Commun. Theor. Phys. 59 (2013) 443 [arXiv:1210.3904] [INSPIRE].

[31] D.E. Kaplan and M. Schmaltz, The little Higgs from a simple group, JHEP 10 (2003) 039 [hep-ph/0302049] [INSPIRE].

[32] M. Schmaltz, The simplest little Higgs, JHEP 08 (2004) 056 [hep-ph/0407143] [INSPIRE].

[33] F. del Aguila, J.I. Illana and M.D. Jenkins, Lepton flavor violation in the simplest little Higgs model, JHEP 03 (2011) 080 [arXiv:1101.2936] [INSPIRE]. 
[34] A. Lami and P. Roig, $H \rightarrow \ell \ell^{\prime}$ in the simplest little Higgs model, Phys. Rev. D 94 (2016) 056001 [arXiv: 1603.09663] [INSPIRE].

[35] CMS collaboration, Search for lepton-flavour-violating decays of the Higgs boson, Phys. Lett. B 749 (2015) 337 [arXiv: 1502.07400] [INSPIRE].

[36] B. Yang, J. Han and N. Liu, Lepton flavor violating Higgs boson decay $h \rightarrow \mu \tau$ in the littlest Higgs model with T parity, Phys. Rev. D 95 (2017) 035010 [arXiv: 1605.09248] [INSPIRE].

[37] S. Chamorro-Solano, A. Moyotl and M.A. Perez, The decay $h \rightarrow \mu \tau$ in the Littlest Higgs Model with T-parity, J. Phys. Conf. Ser. 761 (2016) 012051.

[38] F. del Aguila, Ll. Ametller, J.I. Illana, J. Santiago, P. Talavera and R. Vega-Morales, in preparation.

[39] M. Blanke et al., Rare and CP-violating $K$ and $B$ decays in the littlest Higgs model with $T^{-}$ parity, JHEP 01 (2007) 066 [hep-ph/0610298] [INSPIRE].

[40] A. Denner, Techniques for calculation of electroweak radiative corrections at the one loop level and results for W physics at LEP-200, Fortsch. Phys. 41 (1993) 307 [arXiv:0709.1075] [INSPIRE].

[41] Particle Data Group collaboration, C. Patrignani et al., Review of particle physics, Chin. Phys. C 40 (2016) 100001.

[42] S.R. Coleman and E.J. Weinberg, Radiative corrections as the origin of spontaneous symmetry breaking, Phys. Rev. D 7 (1973) 1888 [inSPIRE].

[43] R. Mertig, M. Böhm and A. Denner, FEYN CALC: computer algebraic calculation of Feynman amplitudes, Comput. Phys. Commun. 64 (1991) 345 [InSPIRE].

[44] V. Shtabovenko, R. Mertig and F. Orellana, New developments in FeynCalc 9.0, Comput. Phys. Commun. 207 (2016) 432 [arXiv:1601.01167] [INSPIRE].

[45] G. Passarino and M.J.G. Veltman, One loop corrections for $e^{+} e^{-}$annihilation into $\mu^{+} \mu^{-}$in the Weinberg model, Nucl. Phys. B 160 (1979) 151 [INSPIRE].

[46] F. del Aguila, M. Pérez-Victoria and J. Santiago, Effective description of quark mixing, Phys. Lett. B 492 (2000) 98 [hep-ph/0007160] [INSPIRE].

[47] K. Agashe and R. Contino, Composite Higgs-mediated FCNC, Phys. Rev. D 80 (2009) 075016 [arXiv:0906.1542] [INSPIRE].

[48] T. Han, H.E. Logan, B. McElrath and L.-T. Wang, Phenomenology of the little Higgs model, Phys. Rev. D 67 (2003) 095004 [hep-ph/0301040] [InSPIRE]. 